\title{
Phylogenetic comparison between Type IX Secretion System (T9SS) protein components suggests evidence of horizontal gene transfer
}

\author{
Reeki Emrizal ${ }^{1}$, Nor Azlan Nor Muhammad ${ }^{\text {Corresp. } 1}$ \\ ${ }^{1}$ Institute of Systems Biology, Universiti Kebangsaan Malaysia, Bangi, Selangor, Malaysia \\ Corresponding Author: Nor Azlan Nor Muhammad \\ Email address: norazlannm@ukm.edu.my
}

Porphyromonas gingivalis is one of the major bacteria that causes periodontitis. Chronic periodontitis is a severe form of periodontal disease that ultimately leads to tooth loss. Virulence factors that contribute to periodontitis are secreted by Type IX Secretion System (T9SS). There are aspects of T9SS protein components that have yet to be characterised. Thus, the aim of this study is to investigate the phylogenetic relationship between members of 20 T9SS component protein families. The Bayesian Inference (BI) trees for 19 T9SS protein components exhibit monophyletic clades for all major classes under Bacteroidetes with strong support for the monophyletic clades or its subclades that is consistent with phylogeny exhibited by the constructed BI tree of 16S rRNA. The BI tree of PorR is different from the $19 \mathrm{BI}$ trees of T9SS protein components as it does not exhibit monophyletic clades for all major classes under Bacteroidetes. There is strong support for the phylogeny exhibited by the BI tree of PorR which deviates from the phylogeny based on 16S rRNA. Hence, it is possible that the porR gene is subjected to horizontal transfer as it is known that virulence factor genes could be horizontally transferred. Seven genes (porR included) that are involved in the biosynthesis of A-LPS are found to be flanked by insertion sequences (IS5 family transposons). Therefore, the intervening DNA segment that contains the porR gene might be transposed and subjected to conjugative transfer. Thus, the seven genes can be co-transferred via horizontal gene transfer. The BI tree of UgdA does not exhibit monophyletic clades for all major classes under Bacteroidetes which is similar to the BI tree of PorR (both are a part of the seven genes). Both BI trees also exhibit similar topology as the four identified clusters with strong support and have similar relative positions to each other in both $\mathrm{BI}$ trees. This reinforces the possibility that porR and the other six genes might be horizontally transferred. Other than the BI tree of PorR, the 19 other BI trees of T9SS protein components also exhibit evidence of horizontal gene transfer. However, their genes might undergo horizontal gene transfer less frequently compared to porR because the intervening DNA segment that contains porR is easily Peer] reviewing PDF | (2019:06:38397:4:0:NEW 27 Mar 2020) 
exchanged between bacteria under Bacteroidetes due to the presence of insertion sequences (IS5 family transposons) that flank it. In conclusion, this study can provide a better understanding about the phylogeny of T9SS protein components. 


\section{Phylogenetic comparison between Type IX Secretion}

2 System (T9SS) protein components suggests

3 evidence of horizontal gene transfer

Reeki Emrizal ${ }^{1}$, Nor Azlan Nor Muhammad $^{1}$

${ }^{1}$ Centre for Bioinformatics Research, Institute of Systems Biology (INBIOSIS), Universiti Kebangsaan Malaysia, 43600 UKM Bangi, Selangor Darul Ehsan, Malaysia

Corresponding Author:

Nor Azlan Nor Muhammad ${ }^{1}$

Centre for Bioinformatics Research, Institute of Systems Biology (INBIOSIS), Universiti

Kebangsaan Malaysia, 43600 UKM Bangi, Selangor Darul Ehsan, Malaysia

Email address: norazlannm@ukm.edu.my 


\section{Abstract}

38

39

40

41

42

43

44

45

46

47

48

49

50

51

52

53

54

55

56

57

58

59

60

61

62

63

64

65

66

67

68

69

70

71

72

73

74

75
Porphyromonas gingivalis is one of the major bacteria that causes periodontitis. Chronic periodontitis is a severe form of periodontal disease that ultimately leads to tooth loss. Virulence factors that contribute to periodontitis are secreted by Type IX Secretion System (T9SS). There are aspects of T9SS protein components that have yet to be characterised. Thus, the aim of this study is to investigate the phylogenetic relationship between members of 20 T9SS component protein families. The Bayesian Inference (BI) trees for 19 T9SS protein components exhibit monophyletic clades for all major classes under Bacteroidetes with strong support for the monophyletic clades or its subclades that is consistent with phylogeny exhibited by the constructed BI tree of $16 \mathrm{~S}$ rRNA. The BI tree of PorR is different from the $19 \mathrm{BI}$ trees of T9SS protein components as it does not exhibit monophyletic clades for all major classes under Bacteroidetes. There is strong support for the phylogeny exhibited by the BI tree of PorR which deviates from the phylogeny based on 16S rRNA. Hence, it is possible that the porR gene is subjected to horizontal transfer as it is known that virulence factor genes could be horizontally transferred. Seven genes (porR included) that are involved in the biosynthesis of A-LPS are found to be flanked by insertion sequences (IS5 family transposons). Therefore, the intervening DNA segment that contains the porR gene might be transposed and subjected to conjugative transfer. Thus, the seven genes can be co-transferred via horizontal gene transfer. The BI tree of UgdA does not exhibit monophyletic clades for all major classes under Bacteroidetes which is similar to the BI tree of PorR (both are a part of the seven genes). Both BI trees also exhibit similar topology as the four identified clusters with strong support and have similar relative positions to each other in both $\mathrm{BI}$ trees. This reinforces the possibility that porR and the other six genes might be horizontally transferred. Other than the BI tree of PorR, the 19 other BI trees of T9SS protein components also exhibit evidence of horizontal gene transfer. However, their genes might undergo horizontal gene transfer less frequently compared to por $R$ because the intervening DNA segment that contains por $R$ is easily exchanged between bacteria under Bacteroidetes due to the presence of insertion sequences (IS5 family transposons) that flank it. In conclusion, this study can provide a better understanding about the phylogeny of T9SS protein components. 


\section{Introduction}

77

78

79

80

81

82

83

84

85

86

87

88

89

90

91

92

93

94

95

96

97

98

99

100

101

102

103

104

105

106

107

108

109

110

111

112

113

114

115
Periodontitis is a form of periodontal disease that is driven by the inflammatory conditions that have deteriorating effects on the structures that support the teeth, including gingiva (gum), alveolar bone, and periodontal ligament. Prolonged inflammatory conditions in chronic periodontitis can cause the destruction of those supporting structures that ultimately leads to tooth loss and might contribute to systemic inflammation (Kinane, Stathopoulou \& Papapanou, 2017; Escobar et al., 2018). This is evidenced by its implications in systemic diseases such as atherosclerosis (Gotsman et al., 2007), aspiration pneumonia (Benedyk et al., 2016), cancer (Gao et al., 2016), rheumatoid arthritis (Laugisch et al., 2016), and diabetes mellitus (Khader et al., 2006). Porphyromonas gingivalis is an oral pathogen that is frequently associated with periodontitis and it is found to acquire Type IX Secretion System (T9SS); a bacterial secretion system that is unique to gram-negative bacteria under the Bacteroidetes phylum (Sato et al., 2010).

T9SS exhibits diverse roles among species of bacteria under Bacteroidetes. Other than transporting virulence factors such as gingipains and peptidylarginine deiminase in $P$. gingivalis that can cause human oral diseases (Potempa, Pike \& Travis, 1995; Maresz et al., 2013), T9SS also transports virulence factors such as chondroitin sulfate lyases that can cause columnaris disease which is a form of fish disease. Flavobacterium columnare, a fish pathogen that contributes to the epidemic that occurred among wild and cultured fish, is found to acquire T9SS. This epidemic poses a problem to the aquaculture industry as columnaris disease can significantly increase the mortality rate among cultured fish, thus threatening the industry output (Li et al., 2017). T9SS is also involved in the transport of non-virulence factors such as cargo proteins that form the bacterial gliding motility apparatus in Flavobacterium johnsoniae that aids in its motility (Nakane et al., 2013) and enzymes that are important for lignocellulose digestion in the rumen of ruminants that become the hosts for Candidatus Paraporphyromonas polyenzymogenes (Naas et al., 2018).

Gram-negative bacteria have an outer membrane (OM) that acts as an impermeable layer that prevents the free movement of hydrophilic and hydrophobic molecules across it. This is because of the presence of lipopolysaccharides (LPS) within the outer leaflet of the OM. Outer membrane proteins that are embedded in the OM usually form a channel to allow small molecules to pass through it (Nikaido, 2003; Hong et al., 2006). However, large molecules such as proteins require larger channels to pass through the OM. Hence, secretion systems are developed by bacteria to enable coordinated transport of specific cargo proteins across the OM. Currently, there are nine different types of secretion systems evolved by bacteria. T9SS is restricted to bacteria under Bacteroidetes (Sato et al., 2010; Lasica et al., 2017).

T9SS consists of many different protein components that perform coordinated roles to ensure proper translocation and modification of its cargo proteins. These roles can be categorised into four major functions: translocation, modification, energetic, and regulation (Sato et al., 2010; Lasica et al., 2017; Naito et al., 2019). Initially, the cargo proteins of T9SS are 
116 translocated across the inner membrane (IM) via Sec translocon where the signal peptide (SP) of 117 cargo proteins is cleaved (Rahman et al., 2003). The cargo proteins also acquire a C-terminal 118 domain (CTD) that interacts with the PorK $_{2} \mathrm{~L}_{3} \mathrm{M}_{2} \mathrm{~N}_{2}$ trans-envelope complex to translocate cargo 119 proteins across the periplasm (Vincent et al., 2017; Vincent, Chabalier \& Cascales, 2018) (Fig.

120

121

122

123

124

125

126

127

128

129

130

131

132

133

134

135

136

137

138

139

140

141

142

143

144

145

146

147

148

149

150

151

152

153

154

1). PorE has been suggested to form the scaffold of the periplasm complex that translocates cargo proteins across the periplasm (Heath et al., 2016; Naito et al., 2019). SprA (ortholog of Sov in $F$. johnsoniae) has been proposed as the secretion pore that translocates cargo proteins across the OM (Lauber et al., 2018). PorV acts as an outer membrane shuttle protein that delivers the cargo proteins to the attachment complex (Glew et al., 2017) (Fig. 1). In the attachment complex, PorU cleaves the CTD of cargo protein. Then, it is glycosylated with anionic lipopolysaccharide (A-LPS) delivered by PorZ at the cleaved site (Glew et al., 2012, 2017). After both post-translational modifications, the cargo protein will be anchored to the cell surface by ALPS (Lasica et al., 2016; Glew et al., 2017) (Fig. 1). PorX and PorY forms a two-component system (TCS) that regulates the operon of por genes (porP, porK, porL, porM, and $\operatorname{por} N$ ) via SigP (Vincent et al., 2017; Kadowaki et al., 2016) (Fig. 1). PorR is an aminotransferase that is involved in the Wbp pathway that biosynthesises the structural repeating unit of anionic polysaccharide (APS) (Shoji et al., 2002; Shoji et al., 2014) (Fig. 1). Despite that, there are T9SS components without known functions (PorP, PorT, PorW, Omp17, PorF, and PorG) (Fig. 1) and a few aspects of T9SS components that have yet to be characterised (Nguyen et al., 2009; Saiki \& Konishi, 2010; Sato et al., 2010; Gorasia et al., 2016; Naito et al., 2019; Taguchi et al., 2016).

This work aims to characterise the phylogeny of T9SS protein components. Phylogenetic analysis was performed on the members of 20 T9SS component protein families that have been reported (Emrizal \& Muhammad, 2018). The Bayesian Inference (BI) trees for 19 T9SS protein components exhibit monophyletic clades for all major classes under Bacteroidetes with strong support for the monophyletic clades or its subclades that is consistent with phylogeny exhibited by the constructed BI tree of $16 \mathrm{~S}$ rRNA. The BI tree of PorR is different from the other $19 \mathrm{BI}$ trees as it does not exhibit monophyletic clades for all major classes under Bacteroidetes. There is also strong support for the phylogeny exhibited by the BI tree of PorR. Thus, there is a possibility that the porR gene is subjected to horizontal transfer as it is known that virulence factor genes could be horizontally transferred (Hirt, Schlievert \& Dunny, 2002). Seven genes including porR that are involved in the biosynthesis of A-LPS are found to be flanked by insertion sequences (IS5 family transposons). This suggests that the intervening DNA segment that contains porR can be transposed and subjected to conjugative transfer (Thomas \& Nielsen, 2005; Brochet et al., 2009). Thus, the seven genes might be co-transferred via horizontal gene transfer. The BI trees of PorR and UgdA (both are a part of the seven genes) exhibit similarities. This reinforces the possibility that porR and the other six genes might undergo horizontal gene transfer. Other than the BI tree of PorR, the BI trees of the other 19 components also exhibit evidence of horizontal gene transfer. However, for the genes that encode those 19 components, they might undergo horizontal gene transfer less frequently compared to porR because the

Peer) reviewing PDF | (2019:06:38397:4:0:NEW 27 Mar 2020) 
155 intervening DNA segment that contains por $R$ is easily exchanged between bacteria under

156 Bacteroidetes due to the presence of IS5 family transposons that flank it.

\section{Materials \& Methods}

158

159

160

161

162

163

164

165

166

167

168

169

170

171

172

173

174

175

176

177

178

179

180

181

182

183

184

185

186

187

188

189

190

191

192

193

\section{Construction of multiple sequence alignments of T9SS protein components}

The multiple sequence alignments for each T9SS protein component were built using the putative members of T9SS component protein families. The pipeline that was used to select those members has been reported (Emrizal \& Muhammad, 2018). The pipeline was used to filter out false positives among the homologs that have been identified through homology searching using BLASTP which was performed using T9SS component protein sequences retrieved from the NCBI protein database that were searched against a local BLAST database constructed from completely sequenced bacterial proteomes from GenBank. The selection criteria used in the pipeline (e-value $\leq 0.001$, query coverage $>60 \%$, and Bacteroidetes homolog with the lowest evalue for bacterial strains with multiple hits) can minimise the possibility of false positive inclusion (Emrizal \& Muhammad, 2018). The sequences of protein homologs used to build the multiple sequence alignments for each T9SS component were provided in FASTA format as Supplemental Information (Data S1).

The multiple sequence alignments were constructed using MAFFT (version 7.402) (Katoh et al., 2002) on the CIPRES computing cluster (Miller, Pfeiffer \& Schwartz, 2010) in FASTA format. Unreliable alignment regions in the multiple sequence alignments were assessed using Guidance 2 (version 2.02) (Sela et al., 2015) on the CIPRES computing cluster (Miller, Pfeiffer \& Schwartz, 2010). Columns with low confidence were removed from the multiple sequence alignments. The format of multiple sequence alignments was converted into relaxed interleaved PHYLIP format using an online Format Converter (https://www.hiv.lanl.gov/content/sequence/FORMAT_CONVERSION/form.html). The multiple sequence alignments in relaxed interleaved PHYLIP format were manually edited into NEXUS format.

\section{Determination of amino acid substitution models for multiple sequence alignments of T9SS protein components}

The multiple sequence alignments in relaxed interleaved PHYLIP format (Data S2) were used by ProtTest (version 3.4.2) (Guindon \& Gascuel, 2003; Darriba et al., 2011) to determine the amino acid substitution model to be used for each alignment in the phylogenetic analysis. The graphical user interface (GUI) version of ProtTest was used to test each alignment against 10 amino acid substitution model matrices (Blosum62, CpREV, Dayhoff, JTT, MtMam, MtREV, RtREV, VT, WAG, and LG) with any combination of among-site rate variation (no rate variation across sites, gamma-shaped rate variation across sites $(+\mathrm{G})$, a proportion of invariable sites $(+\mathrm{I})$, or gammashaped rate variation across sites with a proportion of invariable sites $(+\mathrm{G}+\mathrm{I}))$ and stationary amino acid frequencies (Dirichlet or fixed (empirical) $(+\mathrm{F})$ ). The best model according to 
194 Bayesian Information Criterion (BIC) (Schwarz, 1978) was selected to be used in the

195

196

197

198

199

200

201

202

203

204

205

206

207

208

209

210

211

212

213

214

215

216

217

218

219

220

221

222

223

224

225

226

227

228

229

230

231

232

233

phylogenetic analysis for that alignment.

\section{Bayesian Inference (BI) analysis for multiple sequence alignments of T9SS protein components}

Bayesian Inference (BI) analysis was performed using multiple sequence alignments in NEXUS format (Data S3). The BI analysis was performed using MrBayes (version 3.2.6) (Huelsenbeck \& Ronquist, 2001) on the CIPRES computing cluster (Miller, Pfeiffer \& Schwartz, 2010) for alignments of 14 components (PorK, PorL, PorM, PorN, PorP, PorQ, PorT, PorU, PorV, SigP, Omp17, PorE, PorF, and PorG). The BI analysis for each alignment was performed with the selected amino acid substitution model and two independent runs for 50,000,000 generations, each with four chains, with a sampling frequency of every 5,000 , and a burn-in of $25 \%$. Beagle CPU was utilised to speed up the BI analysis.

The BI analysis for the other 6 components (PorR, Sov, PorW, PorX, PorY, and PorZ) was performed using command-line MrBayes (version 3.2.6) (Huelsenbeck \& Ronquist, 2001) on a desktop with Nvidia Titan V GPU and CUDA driver (version 10.1) installed. The BI analysis for each alignment was performed with the selected amino acid substitution model and two independent runs for 50,000,000 generations, each with four chains (PorR, Sov, PorW) or eight chains (PorX, PorY, and PorZ), with a sampling frequency of every 5,000, and a burn-in of $25 \%$. Beagle GPU was utilised to speed up the BI analysis. The constructed BI trees were visualised and annotated using online iTOL (version 4.4.2) (Letunic \& Bork, 2019).

\section{Construction of Bayesian Inference (BI) tree of 16S ribosomal RNA (rRNA)}

The 16S ribosomal RNA (rRNA) sequences have been used to construct the current universal tree of life (Winker \& Woese, 1991; Pylro et al., 2012). Thus, the BI tree of 16S rRNA has been constructed in this work to compare it with the BI trees of T9SS protein components. A preformatted BLAST database of microbial 16S rRNA sequences was retrieved from NCBI (ftp://ftp.ncbi.nlm.nih.gov/blast/db/). The 16S rRNA sequence from Porphyromonas gingivalis ATCC 33277 (NR_040838.1) was retrieved from NCBI (https://www.ncbi.nlm.nih.gov/gene) and it was searched against that database using local BLASTN (Altschul et al., 1990). The pipeline mentioned above was used to select homologs of 16S rRNA gene in species under Bacteroidetes that were also found to acquire homologs of T9SS protein components in this work. For those species that their 16S rRNA sequences could not be retrieved from the microbial 16S rRNA BLAST database, their 16S rRNA sequences were retrieved directly from either NCBI Gene (https://www.ncbi.nlm.nih.gov/gene) or NCBI Nucleotide (https://www.ncbi.nlm.nih.gov/ nuccore/). The selected 16S rRNA sequences were provided in FASTA format as a Supplemental Information (Data S1).

The sequences were used to build the multiple sequence alignment of $16 \mathrm{~S}$ rRNA using MAFFT (version 7.402) (Katoh et al., 2002) and unreliable alignment regions in the multiple sequence alignment were assessed using Guidance 2 (version 2.02) (Sela et al., 2015) on the 
234 CIPRES computing cluster (Miller, Pfeiffer \& Schwartz, 2010). Columns with low confidence 235 were removed from the multiple sequence alignment. The alignment in FASTA format (Data S2) 236 was used to determine the best nucleotide substitution model to be used in the phylogenetic 237 analysis. The graphical user interface (GUI) version of ModelTest (Darriba et al., 2019) was used 238 to test the alignment against 3 nucleotide substitution model matrices (GTR, HKY85, and F81) 239 with any combination of among-site rate variation (no rate variation across sites, gamma-shaped

240

241

242

243

244

245

246

247

248

249

250

251

252

253

254

255

256

257

258

259

260

261

262

263

264

265

266

267

268

269

270

271

272

273 rate variation across sites $(+G)$, a proportion of invariable sites $(+I)$, or gamma-shaped rate variation across sites with a proportion of invariable sites $(+\mathrm{G}+\mathrm{I}))$ and stationary amino acid frequencies (Dirichlet or fixed (empirical) $(+\mathrm{F}))$. The best model according to Bayesian Information Criterion (BIC) (Schwarz, 1978) was selected to be used in the phylogenetic analysis for that alignment.

BI analysis was performed using the alignment in NEXUS format (Data S3). The analysis was performed using MrBayes (version 3.2.6) (Huelsenbeck \& Ronquist, 2001) on the CIPRES computing cluster (Miller, Pfeiffer \& Schwartz, 2010) with the selected nucleotide substitution model and two independent runs for 50,000,000 generations, each with four chains, with a sampling frequency of every 5,000 , and a burn-in of $25 \%$. Beagle CPU was utilised to speed up the BI analysis. The BI tree of $16 \mathrm{~S}$ rRNA was visualised and annotated using online iTOL (version 4.4.2) (Letunic \& Bork, 2019).

\section{Identification of porR and its neighbouring genes' arrangement in Porphyromonas gingivalis ATCC 33277 genome}

The sequence of $P$. gingivalis ATCC 33277 genome and annotation files of the genome were retrieved from Genbank (Naito et al., 2008). The $P$. gingivalis ATCC 33277 genome sequence and its annotation files were provided in the Supplemental Information (Data S4). The part of $P$. gingivalis ATCC 33277 genome sequence that contains the por $R$ and its neighbouring genes was extracted. Then, it was searched against the non-redundant protein sequences (nr) database using online BLASTX. The search was narrowed down to the proteome of $P$. gingivalis ATCC 33277 only. The maximum target sequences were set at the highest value available which is 20,000 . Other parameters were left at its default values (Altschul et al., 1990). Only the matches with $100 \%$ percentage identity and 0 e-value were used to annotate the part of $P$. gingivalis ATCC 33277 genome sequence that contains the porR gene.

\section{Construction of Bayesian Inference (BI) tree of UgdA}

Based on the identification of porR neighbouring genes, the two genes that are involved in the Wbp pathway ( $u g d A$ and porR) are found to be within the intervening DNA segment that is flanked by IS5 family transposons. Thus, the BI tree of UgdA was constructed to be compared with the BI tree of PorR. The pipeline mentioned above was used to select homologs of UgdA (Data S1) and construct the multiple sequence alignment of UgdA with low confidence columns being removed. The alignment in relaxed interleaved PHYLIP format (Data S2) was used to determine the best amino acid substitution model. BI analysis was performed using UgdA 
274 alignment in NEXUS format (Data S3). The analysis was performed using command-line 275 MrBayes (version 3.2.6) (Huelsenbeck \& Ronquist, 2001) on a desktop with Nvidia Titan V 276 GPU and CUDA driver (version 10.1) installed with the selected amino acid substitution model 277 and two independent runs for 50,000,000 generations, each with four chains, with a sampling 278 frequency of every 5,000, and a burn-in of $25 \%$. Beagle GPU was utilised to speed up the BI 279 analysis. The constructed BI tree was visualised and annotated using online iTOL (version 4.4.2) 280 (Letunic \& Bork, 2019).

281

\section{Results}

283

284

285

286

287

288

289

290

291

292

293

294

295

296

297

298

299

300

301

302

303

304

305

306

307

308

309

310

311

312

313

\section{Bayesian Inference (BI) trees of T9SS protein components}

Bayesian Inference (BI) trees are constructed from the multiple sequence alignments of putative members of T9SS component protein families that have been reported (Emrizal \& Muhammad, 2018). The characteristics of alignments and the best amino acid substitution model that has been selected for each alignment are shown in Table 1. The selected amino acid substitution model for each alignment defines the parameters that were used for BI analysis for each alignment. The unrooted BI trees of T9SS protein components are shown (Figs. 2-6). The identified monophyletic clades that were formed by terminal nodes that belong to the same class under Bacteroidetes are denoted by solid curves (Figs. 2-6). The monophyletic clades or its subclades with strong support (posterior probability value $>0.95$ ) are denoted by dashed curves (Figs. 2-6).

Out of $20 \mathrm{BI}$ trees of T9SS protein components, only 19 exhibit monophyletic clades for all major classes under Bacteroidetes (Figs. 2-6). Major classes are those with more than five families under the class (Bacteroidia, Cytophagia, and Flavobacteriia) while minor classes are those with less than or equal to five families under the class (Chitinophagia, Sphingobacteriia, Saprospiria, Incertae sedis, and unclassified). Nine of the BI trees (PorK, Sov, PorT, PorV, PorW, PorX, Omp17, PorE, and PorF) exhibit monophyletic clades for all major classes under Bacteroidetes with strong support. Ten of the BI trees (PorL, PorM, PorN, PorP, PorQ, PorU, PorY, PorZ, SigP, and PorG) exhibit strong support for the monophyletic clades or its subclades for all major classes under Bacteroidetes (Figs. 2-6). Despite the presence of PorR homologs from species under Bacteroidia, Cytophagia, and Flavobacteriia, the BI tree of PorR does not exhibit monophyletic clades for all major classes under Bacteroidetes (Fig. 3C). Thus, the BI tree of PorR is different compared to the other $19 \mathrm{BI}$ trees of T9SS protein components that exhibit monophyletic clades for all major classes under Bacteroidetes.

Some of the terminal nodes of the $19 \mathrm{BI}$ trees of T9SS protein components are out of their expected monophyletic clades (Figs. 2-6). The species corresponding to those terminal nodes are listed in Table S1. There are species that frequently have their terminal nodes out of their expected monophyletic clades such as Fluviicola taffensis DSM 16823, bacterium L21-SpiD4, Owenweeksia hongkongensis DSM 17368, and Draconibacterium orientale. The terminal nodes corresponding to $F$. taffensis DSM 16823 are found to be out of their expected monophyletic clades in 14 out of 19 BI trees (except PorK, PorN, PorP, PorU, and SigP). The

Peer) reviewing PDF | (2019:06:38397:4:0:NEW 27 Mar 2020) 
314 terminal nodes corresponding to bacterium L21-Spi-D4 are found to be out of their monophyletic 315 clades in 10 out of 19 BI trees (except PorK, PorL, PorM, Sov, PorT, PorU, PorX, PorY, and 316 PorE). The terminal nodes corresponding to O. hongkongensis DSM 17368 are found to be out 317 of their expected monophyletic clades in 6 out of 19 BI trees (PorM, PorP, PorV, PorY, Omp17, 318 and PorE). The terminal nodes corresponding to $D$. orientale are found to be out of their 319 expected monophyletic clades in 7 out of 19 BI trees (PorN, PorP, PorV, PorW, PorY, SigP, and

320

321

322

323

324

325

326

327

328

329

330

331

332

333

334

335

336

337

338

339

340

341

342

343

344

345

346

347

348

349

350

351

352

353 Omp 17). The $20 \mathrm{BI}$ trees with terminal nodes labelled with their corresponding species and support values for each branch are shown in the Supplemental Information (Figs. S1-S20).

\section{Bayesian Inference (BI) tree of 16S rRNA}

The BI tree of 16S rRNA was constructed from the multiple sequence alignment of 16S rRNA homologs from species that were identified to also acquire T9SS protein homologs. Out of 181 species that acquire T9SS protein homologs, only 16S rRNA sequences from 144 species were able to be retrieved from NCBI. The characteristics of $16 \mathrm{~S}$ rRNA alignment and the best nucleotide substitution model that had been selected for that alignment are shown in Table 1. The unrooted BI tree of $16 \mathrm{~S}$ rRNA is shown in Fig. 7. The identified monophyletic clades that were formed by terminal nodes that belong to the same class under Bacteroidetes are denoted by solid curves (Fig. 7). The monophyletic clades or its subclades with strong support (posterior probability value $>0.95$ ) are denoted by dashed curves (Fig. 7).

The BI tree of 16S rRNA was constructed to be compared to the BI trees of T9SS protein components. The 16S rRNA exhibits monophyletic clades for all major classes under Bacteroidetes with strong support (Fig. 7) similar to the 19 BI trees of T9SS protein components. The 16S rRNA also exhibits monophyletic clades for all minor classes under Bacteroidetes with strong support denoted by 4 monophyletic clades of red, pink, yellow, and orange circles (Fig. 7). None of the $20 \mathrm{BI}$ trees of T9SS protein components exhibit phylogeny of the minor classes that is consistent with the phylogeny exhibited by the 16S rRNA tree (Fig. 2-7). Hence, minor classes are excluded in the comparison between $20 \mathrm{BI}$ trees of T9SS protein components. The BI tree of 16S rRNA with terminal nodes labelled with their corresponding species and support values for each branch are shown in the Supplemental Information (Fig. S21).

\section{Arrangement of por $R$ and its neighbouring genes in $\boldsymbol{P}$. gingivalis ATCC 33277 genome}

As shown in Fig. 8, porR and its neighbouring genes are flanked by IS5 family transposons. The IS5 family transposon (cyan rectangles) encodes IS5 family transposase that cleaves the flanking $12 \mathrm{bp}$ inverted repeats (purple triangles) (Fig. 8). This might suggest the possibility that the intervening DNA segment that contains seven genes that are involved in A-LPS biosynthesis (yellow rectangles) can undergo transposition and is possibly subjected to conjugative transfer (Fig. 8) (Thomas \& Nielsen, 2005; Brochet et al., 2009). porR (PGN_1236) and ugdA (PGN_1243) genes (Fig. 8) have been reported to be involved in the Wbp pathway that is important for the biosynthesis of structural sugar (di-acetylated glucuronic acid) of A-LPS (Shoji et al., 2002; Shoji et al., 2014). porS (PGN_1235) and wzy (PGN_1242) genes (Fig. 8) have been 
354

355

356

357

358

359

360

361

362

363

364

365

366

367

368

369

370

371

372

373

374

375

376

377

378

379

380

381

382

383

384

385

386

387

388

389

390

391

392

393

reported to participate in the assembly of A-LPS in bacterial inner membrane (Shoji et al., 2013). gtfB (PGN_1251) and gtfE (PGN_1240) glycosyltransferase genes (Fig. 8) are important for ALPS biosynthesis while $r f a$ (PGN_1255) glycosyltransferase gene (Fig. 8) is important for the biosynthesis of lipid A-core portion of A-LPS (Shoji et al., 2018).

\section{Bayesian Inference (BI) tree of UgdA}

The BI tree of UgdA was constructed from the multiple sequence alignment of UgdA homologs that were identified using the same pipeline that has been reported to select T9SS protein homologs (Emrizal \& Muhammad, 2018). The characteristics of UgdA alignment and the best amino acid substitution model that had been selected for that alignment are shown in Table 1. The unrooted BI tree of UgdA is shown in Fig. 9B. The unrooted BI tree of PorR is also shown in Fig. 9A to be compared with the BI tree of UgdA. Both BI trees do not exhibit monophyletic clades for all major classes under Bacteroidetes. Both BI trees also exhibit similar topology. Four similar clusters (I, II, III, and IV) were identified between both BI trees. Cluster I consists primarily of terminal nodes from Flavobacteriia and a few terminal nodes from other classes. Cluster II consists of terminal nodes from Porphyromonas, Tannerella, and Parabacteroides genera. Cluster III consists of terminal nodes from Rufibacter and Hymenobacter genera. Cluster IV consists of terminal nodes from Prevotella, Bacteroides, Proteiniphilum, and other genera. The BI tree of UgdA with terminal nodes labelled with their corresponding species and support values for each branch are shown in the Supplemental Information (Fig. S22).

\section{Taxonomic distribution of T9SS protein components}

As shown in the 20 BI trees of T9SS components (Figs. 2-6), only bacteria under Bacteroidia, Flavobacteriia, and Chitinophagia classes acquired the 20 components investigated in this work. The bacteria under Cytophagia class acquired only 19 protein components (except PorN). The bacteria under Saprospiria class acquired only 18 protein components (except PorL and PorG). The bacteria under Sphingobacteriia class acquired only 17 protein components (except PorQ, PorU, and PorZ). The unclassified bacteria acquired only 17 protein components (except PorN, PorU, and PorG). The bacteria under Incertae sedis class acquired only 11 protein components (PorQ, PorR, Sov, PorU, PorV, PorX, PorY, PorZ, SigP, Omp17, and PorF).

The findings in this work are consistent with the taxonomic distribution of T9SS components among bacteria under Bacteroidetes where it has been reported that Bacteroidia, Flavobacteriia, Cytophagia, Sphingobacteriia, and Incertae sedis classes acquired T9SS component homologs (McBride \& Zhu, 2013). However, comparing the reported taxonomic distribution of T9SS components to the findings in this work, we have identified other species under Chitinophagia, Saprospiria, and those that are unclassified that have acquired T9SS component homologs. Those species and T9SS component homologs they acquired are illustrated in Fig. 10. 
394

395

396

397

398

399

400

401

402

403

404

405

406

407

408

409

410

411

412

413

414

415

416

417

418

419

420

421

422

423

424

425

426

427

428

429

430

431

432

\section{Discussion}

The 19 Bayesian Inference (BI) trees of T9SS protein components exhibit monophyletic clades for all major classes under Bacteroidetes with strong support for the monophyletic clades or its subclades (Figs. 2-6). Similar to the 19 BI trees of T9SS protein components, the BI tree of $16 \mathrm{~S}$ rRNA also exhibits monophyletic clades for all major classes under Bacteroidetes with strong support (Fig. 7). 16S rRNA has been extensively used in phylogenetic analysis for the purpose of evolutionary comparison and classification. The reliability of this approach lies on the assumption that the 16S rRNA gene undergoes hierarchical and unidirectional evolution and no gene transfer of 16S rRNA occurs between species (Karlsson et al. 2011). Due to the advantages that the 16S rRNA gene has such as ubiquity in bacterial genomes, being easily sequenced, and widely available in public sequence databases, the current universal tree of life is based on the phylogeny of this gene (Winker \& Woese, 1991; Coutinho et al., 1999; Pylro et al., 2012). That assumption has been challenged due to the presence of multiple copies of 16S rRNA in a bacterial genome and the $16 \mathrm{~S}$ rRNA genes from operons in the same genome are rather distinct which might suggest that such genes might have undergone horizontal gene transfer (Pei et al., 2010; Karlsson et al., 2011). However, the extent of 16S rRNA evolution remains considerably less compared to the other genes in the bacterial genome (Espejo \& Plaza, 2018). Thus, 16S rRNA remains relevant for the purpose of evolutionary comparison and classification.

It is expected that the BI trees of T9SS protein components would exhibit similar phylogeny with the BI tree of $16 \mathrm{~S}$ rRNA. However, the BI trees of T9SS protein components exhibit inconsistent positions of terminal nodes from minor classes among themselves and the phylogeny for the minor classes deviate from the phylogeny exhibited by the BI tree of $16 \mathrm{~S}$ rRNA (Figs. 2-7). Hence, the minor classes under Bacteroidetes are excluded from the comparison between the $20 \mathrm{BI}$ trees of T9SS protein components. This might arise due to insufficient taxa from minor classes provided to construct those BI trees. Hence, the information that is provided is insufficient to fully resolve the phylogeny of minor classes. As more T9SSacquiring species from minor classes are sequenced later on, the phylogeny of T9SS protein components will be more resolved (Alvizu et al., 2018).

Different from the other $19 \mathrm{BI}$ trees of T9SS protein components, the BI tree of PorR does not exhibit monophyletic clades for all major classes under Bacteroidetes (Figs. 2-6). The presence of strong support (posterior probability value $>0.95$ ) as denoted by the black branch leading to the top half of the BI tree of PorR (Fig. 3C) suggests that there is strong support that the phylogeny exhibited by the BI tree of PorR deviates from the phylogeny based on the $16 \mathrm{~S}$ rRNA sequence (Fig. 7). Thus, there is a possibility that the porR gene is subjected to horizontal transfer hence causing deviation from the expected phylogeny (Pylro et al., 2012). Hirt, Schlievert \& Dunny have demonstrated that virulence factors and antibiotic resistance genes could be horizontally transferred (Hirt, Schlievert \& Dunny, 2002). Hence, this suggests 
433 the possibility that the porR gene that encodes one of the virulence factors produced by $P$. 434 gingivalis can be horizontally transferred (Shoji et al., 2002; Shoji et al., 2014).

435

436

437

438

439

440

441

442

443

444

445

446

447

448

449

450

451

452

453

454

455

456

457

458

459

460

461

462

463

464

465

466

467

468

469

470

471

472

The arrangement of porR and its neighbouring genes in the P. gingivalis ATCC 33277 genome was identified in order to support the possibility that por $R$ is horizontally transferred. $P$. gingivalis ATCC 33277 genome was chosen because many gene orthologs that are involved in A-LPS biosynthesis have been identified in this genome (Shoji et al., 2018), porR and its neighbouring genes are found to be flanked by insertion sequences (IS5 family transposons) (Fig. 8). The IS5 family transposons (cyan rectangles) contain a single open reading frame that encodes for IS5 family transposase that cleaves the $12 \mathrm{bp}$ inverted repeats (purple triangles) that flank the insertion sequences (Fig. 8). The $12 \mathrm{bp}$ inverted repeats show imperfect homology to each other with the consensus sequence: GAGACCTTTG[CA]A. Both of the IS5 family transposons are $\sim 1300 \mathrm{bp}$ in length. These features are typical of IS5 family transposons (Mahillon \& Chandler, 1998; Naito et al., 2008). The intervening DNA segment and both IS5 family transposons that flank it might form a composite transposon where the cleaving action of IS5 family transposases on inverted repeats can mobilise the intervening DNA segment that contains the por $R$ gene and possibly subject it to conjugative transfer (Thomas $\&$ Nielsen, 2005; Brochet et al., 2009). The length of the composite transposon is $\sim 70 \mathrm{kbp}$. However, it is also possible for IS5 family transposase to cleave the inverted repeat directly downstream of $r f a$ (PGN_1255) (Fig. 8) which will reduce the length of the composite transposon to $\sim 47 \mathrm{kbp}$. It has been reported that a transposon of $\sim 47 \mathrm{kbp}$ in length is able to undergo both transposition and conjugation processes (Brochet et al., 2009). Hence, it might be possible for composite transposons of such length to undergo transposition and subsequently be horizontally transferred via bacterial conjugation.

The intervening DNA segment contains seven genes that are involved in the biosynthesis of A-LPS (Fig. 8). Both porR (PGN_1236) and ugdA (PGN_1243) genes are involved in the Wbp pathway that is important for the biosynthesis of di-acetylated glucuronic acid which is the structural sugar of A-LPS (Shoji et al., 2002; Shoji et al., 2014). The porS gene (PGN_1235), which is an O-antigen flippase, and wzy gene (PGN_1242), which is an O-antigen polymerase, are involved in the assembly of A-LPS on the periplasmic side of bacterial IM (Shoji et al., 2013). gtfB (PGN_1251) and gtfE (PGN_1240) glycosyltransferase genes are involved in the biosynthesis of the sugar moiety of A-LPS. $r f a$ (PGN_1255) glycosyltransferase gene is involved in the biosynthesis of the lipid A-core moiety of A-LPS (Shoji et al., 2018). However, there are other genes that are involved in the biosynthesis of A-LPS and they are spread out throughout the genome (Shoji et al., 2018). Usually, genes that are co-regulated and involved in a similar pathway are clustered in a single operon (Yanofsky \& Lennox, 1959; Osbourn \& Field, 2009). Thus, it is possible that the other genes do not form a cluster with the seven genes that are identified to be flanked by insertion sequences (IS5 family transposons) because they are not coregulated.

It is possible that those seven genes might be co-transferred via horizontal gene transfer. Thus, phylogenetic analysis was performed for the protein alignment of UgdA that is encoded by 
$473 u g d A$ which, together with porR, are involved in the Wbp pathway and are co-localised in the 474 intervening DNA segment flanked by IS5 family transposons (Fig. 8). The BI tree of UgdA (Fig. 475 9B) was constructed to be compared with the BI tree of PorR (Fig. 9A). Different to the 19 BI 476 trees of T9SS protein components, both BI trees do not exhibit monophyletic clades for all major 477 classes under Bacteroidetes. They also exhibit similar topology where four similar clusters (I, II, 478 III, and IV) with strong support (denoted by a black branch leading to the cluster) have been 479 identified in both BI trees. Cluster I consists of terminal nodes from Flavobacteriia and a few 480 terminal nodes from other classes. Cluster II consists of terminal nodes from Porphyromonas, 481 Tannerella, and Parabacteroides genera. Cluster III consists of terminal nodes from Rufibacter 482 and Hymenobacter genera. Cluster IV consists of terminal nodes from Prevotella, Bacteroides, 483 Proteiniphilum, and other genera. These four clusters exhibit similar relative positions to each 484 other in both BI trees (e.g. cluster I is closer to cluster II than the other clusters and cluster III is 485 closer to cluster II than the other clusters). However, due to the differences in branch lengths between both BI trees, they look slightly different as the upper part of the UgdA tree (Fig. 9B) appears more elongated than the upper part of the PorR tree (Fig. 9A), while the lower part of the UgdA tree (cluster I) appears more shortened than the lower part of the PorR tree (cluster I).

Other than the BI tree of PorR, the BI trees of the other 19 T9SS protein components also exhibit evidence of horizontal gene transfer perhaps between classes under Bacteroidetes. As listed in Table S1, there are terminal nodes that are out of their expected monophyletic clades in the BI trees of those components that suggests the genes that encode them might be horizontally transferred. In theory, the common ancestral species of a monophyletic clade for a class under Bacteroidetes passes the gene that encodes T9SS protein components to its descendant species. Thus, the descendant species that are out of their expected monophyletic clades most likely acquired that gene from the common ancestral species of a monophyletic clade from another class that could be interpreted as a horizontal gene transfer between classes under Bacteroidetes (Thomas \& Nielsen, 2005; Brochet et al., 2009). It is interesting to highlight that there are species that frequently have their corresponding terminal nodes in those $19 \mathrm{BI}$ trees out of their expected monophyletic clades (Figs. 2-6) such as F. taffensis DSM 16823, bacterium L21-SpiD4, O. hongkongensis DSM 17368, and D. orientale. Thus, it is likely that those bacteria frequently acquire their T9SS components through horizontal gene transfer. However, for the genes that encode those 19 components, they might undergo horizontal gene transfer less frequently compared to porR that causes most of the terminal nodes of BI trees of those components to cluster according to their respective classes. It might be because the intervening DNA segment that contains the porR gene is easily exchanged between bacteria under Bacteroidetes due to the presence of insertion sequences (IS5 family transposons) that flank it (Fig. 8).

T9SS is made up of various protein components that form the regulation, translocation, energetic, and modification components. Currently, the secretion system is primarily found in bacteria under the Bacteroidetes phylum (Abby et al., 2016). Bacteria from classes under Bacteroidetes (Bacteroidia, Flavobacteriia, Cytophagia, Chitinophagia, Sphingobacteriia, 
513 Saprospiria, Incertae sedis, and unclassified) are found to acquire T9SS protein components

514 (Figs. 2-6). However, not all of them acquire the 20 components that have been reported (Sato et

515 al., 2010; Lasica et al., 2017). As shown in the 20 BI trees of T9SS protein components (Figs. 2-

516 6), only bacteria under Bacteroidia, Flavobacteriia, and Chitinophagia acquired the 20

517 components investigated. The bacteria under Cytophagia only acquired 19 components (except

518 PorN). The bacteria under Saprospiria only acquired 18 components (except PorL and PorG).

519 The bacteria under Sphingobacteriia only acquired 17 components (except PorQ, PorU, and

520 PorZ). The unclassified bacteria only acquired 17 components (except PorN, PorU, and PorG).

521 The bacteria under Incertae sedis only acquired 11 components (PorQ, PorR, Sov, PorU, PorV,

522 PorX, PorY, PorZ, SigP, Omp17, and PorF). It is interesting to note that PorU, PorZ, and PorQ

523 form the modification components of T9SS. Thus, Sphingobacteriia does not acquire the

524 components that perform post-translational modifications on T9SS cargo proteins such as

525 cleavage of CTD and A-LPS glycosylation. Perhaps, T9SS acquired by Sphingobacteriia does

526 not cleave the CTD of cargo protein and glycosylate it with A-LPS, but leaves the cargo protein

527 bounded to PorV after it is translocated to bacterial cell surface by Sov. Another possible

528 explanation is that Sphingobacteriia does have proteins that perform the functions of missing

529 protein components. However, those proteins exhibit limited sequence similarity with any

530 currently known T9SS protein component. Thus, they could not be detected by the homology

531 searching method used in this work. This explanation could also be applied for other species of

532 bacteria under Bacteroidetes that do not acquire the homologs of the 20 T9SS components.

533 This work has found other species under Chitinophagia, Saprospiria, and those that are

534 unclassified that acquired homologs of T9SS components that, to our knowledge, might not have

535 been reported (McBride \& Zhu, 2013). Those other species and the homologs of T9SS

536 components they acquired are shown in Fig. 10. This identification might be due to the analysis

537 that was performed which might cover more bacterial species than previous works as more

538 bacterial genomes have been completely sequenced in the past few years.

539

540 Conclusions

541

542 The objective of this work was to investigate the phylogenetic relationship among putative

543 members of 20 T9SS component protein families (Emrizal \& Muhammad, 2018). The Bayesian

544 Inference (BI) trees for 19 T9SS protein components exhibit monophyletic clades for all major

545 classes under Bacteroidetes with strong support for the monophyletic clades or its subclades,

546 which is consistent with the phylogeny exhibited by the constructed BI tree of 16S rRNA.

547 However, the BI tree of PorR is different from the other 19 BI trees of T9SS protein components

548 as it does not exhibit monophyletic clades for all major classes under Bacteroidetes. There is

549 strong support for the phylogeny exhibited by the BI tree of PorR which deviates from the

550 phylogeny based on the 16S rRNA sequence. Thus, there is a possibility that the porR gene is

551 subjected to horizontal transfer as it is known that virulence factor genes could be horizontally

552 transferred. Seven genes that are involved in the biosynthesis of A-LPS that includes por $R$ are 
553 found to be flanked by insertion sequences (IS5 family transposons). This suggests that the

554

555

556

557

558

559

560

561

562

563

564

565

566

567

568

569

570

571

572

573

574

575

576

577

578

579

580

581

582

583

584

585

586

587

588

589

590

591

592

593

594

intervening DNA segment that contains the porR gene can be transposed and subjected to conjugative transfer. Thus, the seven genes might be co-transferred via horizontal gene transfer. Similar to the BI tree of PorR, the BI tree of UgdA does not exhibit monophyletic clades for all major classes under Bacteroidetes (both are a part of the seven genes). Both BI trees also exhibit similar topology where the four identified clusters with strong support have similar relative positions to each other in both BI trees. Other than the BI tree of PorR, the BI trees of the other 19 components also exhibit evidence of horizontal gene transfer. However, for the genes that encode those 19 components, they might undergo horizontal gene transfer less frequently compared to porR because the intervening DNA segment that contains porR is easily exchanged between bacteria under Bacteroidetes due to the presence of insertion sequences (IS5 family transposons) that flank it. This work also found other species under Chitinophagia, Saprospiria, and those that are unclassified that acquired T9SS component protein homologs that, to our knowledge, might not have been reported.

\section{Acknowledgements}

The authors acknowledge Universiti Kebangsaan Malaysia (UKM) grant, DPP-2018-010 for providing funds to support this work. We gratefully acknowledge the support of NVIDIA Corporation with the donation of the Titan V GPU used for this research. We thank the Centre for Bioinformatics Research (CBR) for providing the facilities to conduct the bioinformatics analyses. We thank the reviewers for their comments on previous drafts of the manuscript.

\section{References}

Abby SS, Cury J, Guglielmini J, Néron B, Touchon M, Rocha EPC. 2016. Identification of protein secretion systems in bacterial genomes. Scientific Reports 6:23080 DOI 10.1038/srep23080.

Altschul SF, Gish W, Miller W, Myers EW, Lipman DJ. 1990. Basic local alignment search tool. Journal of Molecular Biology 215(3):403-410 DOI 10.1016/S0022-2836(05)80360-2.

Alvizu A, Eilertsen MH, Xavier JR, Rapp HT. 2018. Increased taxon sampling provides new insights into the phylogeny and evolution of the subclass Calcaronea (Porifera, Calcarea). Organisms Diversity and Evolution 18(3):279-290 DOI 10.1007/s13127-018-0368-4.

Benedyk M, Mydel PM, Delaleu N, Plaza K, Gawron K, Milewska A, Maresz K, Koziel J, Pyrc K, Potempa J. 2016. Gingipains: critical factors in the development of aspiration pneumonia caused by Porphyromonas gingivalis. Journal of Innate Immunity 8(2):185-198 DOI 10.1159/000441724.

Brochet M, Da Cunha V, Couvé E, Rusniok C, Trieu-Cuot P, Glaser P. 2009. Atypical association of DDE transposition with conjugation specifies a new family of mobile elements. Molecular Microbiology 71(4):948-959 DOI 10.1111/j.1365-2958.2008.06579.x.

Coutinho HL, De VO, Manfio GP, Rosado AS. 1999. Evaluating the microbial diversity of soil samples: methodological innovations. Anais da Academia Brasileira de Ciências 71(3 Pt

Peer) reviewing PDF | (2019:06:38397:4:0:NEW 27 Mar 2020) 
595

596

597

598

599

600

601

602

603

604

605

606

607

608

609

610

611

612

613

614

615

616

617

618

619

620

621

622

623

624

625

626

627

628

629

630

631

632

633

634

635

636

637

638

639

640

2):491-503

Darriba D, Taboada GL, Doallo R, Posada D. 2011. ProtTest 3: fast selection of best-fit models of protein evolution. Bioinformatics 27(8):1164-1165 DOI 10.1093/bioinformatics/btr088.

Darriba D, Posada D, Kozlov AM, Stamatakis A, Morel B, Flouri T. 2019. ModelTest-NG: A New and Scalable Tool for the Selection of DNA and Protein Evolutionary Models. Molecular Biology and Evolution:msz189 DOI 10.1093/molbev/msz189.

Emrizal R, Muhammad NAN. 2018. Identification of sequence motifs for the protein components of type IX secretion system. Sains Malaysiana 47(12):2941-2950 DOI 10.17576/jsm-2018-4712-02.

Escobar GF, Abdalla DR, Beghini M, Gotti VB, Junior VR, Napimoga MH, Ribeiro BM, Rodrigues DBR, Nogueira RD, de Lima Pereira SA. 2018. Levels of pro and antiinflammatory citokynes and c-reactive protein in patients with Chronic Periodontitis submitted to nonsurgical periodontal treatment. Asian Pacific Journal of Cancer Prevention : APJCP 19(7):1927-1933 DOI 10.22034/APJCP.2018.19.7.1927.

Espejo RT, Plaza N. 2018. Multiple Ribosomal RNA Operons in Bacteria; Their Concerted Evolution and Potential Consequences on the Rate of Evolution of Their 16S rRNA. Frontiers in Microbiology 9:1232 DOI 10.3389/fmicb.2018.01232.

Gao S, Li S, Ma Z, Liang S, Shan T, Zhang M, Zhu X, Zhang P, Liu G, Zhou F, Yuan X, Jia R, Potempa J, Scott DA, Lamont RJ, Wang H, Feng X. 2016. Presence of Porphyromonas gingivalis in esophagus and its association with the clinicopathological characteristics and survival in patients with esophageal cancer. Infectious Agents and Cancer 11(1):3 DOI 10.1186/s13027-016-0049-X.

Glew MD, Veith PD, Chen D, Gorasia DG, Peng B, Reynolds EC. 2017. PorV is an outer membrane shuttle protein for the type IX secretion system. Scientific Reports 7(1):8790 DOI 10.1038/s41598-017-09412-w.

Glew MD, Veith PD, Peng B, Chen YY, Gorasia DG, Yang Q, Slakeski N, Chen D, Moore C, Crawford S, Reynolds EC. 2012. PG0026 is the C-terminal signal peptidase of a novel secretion system of Porphyromonas gingivalis. Journal of Biological Chemistry 287(29):24605-24617 DOI 10.1074/jbc.M112.369223.

Gorasia DG, Veith PD, Hanssen EG, Glew MD, Sato K, Yukitake H, Nakayama K, Reynolds EC. 2016. Structural insights into the PorK and PorN components of the Porphyromonas gingivalis type IX secretion system. PLoS Pathogens 12(8):e1005820 DOI 10.1371/journal.ppat.1005820.

Gotsman I, Lotan C, Soskolne WA, Rassovsky S, Pugatsch T, Lapidus L, Novikov Y, Masrawa S, Stabholz A. 2007. Periodontal destruction is associated with coronary artery disease and periodontal infection with acute coronary syndrome. Journal of Periodontology 78(5):849-858 DOI 10.1902/jop.2007.060301.

Guindon S, Gascuel O. 2003. A simple, fast, and accurate algorithm to estimate large phylogenies by maximum likelihood. Systematic Biology 52(5):696-704 DOI 10.1080/10635150390235520.

Heath JE, Seers CA, Veith PD, Butler CA, Muhammad NAN, Chen YY, Slakeski N, Peng B, Zhang L, Dashper SG, Cross KJ, Cleal SM, Moore C, Reynolds EC. 2016. PG1058 is a novel multidomain protein component of the bacterial type IX secretion system. PloS One 11(10):e0164313 DOI 10.1371/journal.pone.0164313.

Hirt H, Schlievert PM, Dunny GM. 2002. In vivo induction of virulence and antibiotic

Peer) reviewing PDF | (2019:06:38397:4:0:NEW 27 Mar 2020) 
641

642

643

644

645

646

647

648

649

650

651

652

653

654

655

656

657

658

659

660

661

662

663

664

665

666

667

668

669

670

671

672

673

674

675

676

677

678

679

680

681

682

683

684

685

686

resistance transfer in Enterococcus faecalis mediated by the sex pheromone-sensing system of pCF10. Infection and Immunity 70(2):716-723 DOI 10.1128/IAI.70.2.716.

Hong H, Patel DR, Tamm LK, Van Den Berg B. 2006. The outer membrane protein OmpW forms an eight-stranded $\beta$-barrel with a hydrophobic channel. Journal of Biological Chemistry 281(11):7568-7577 DOI 10.1074/jbc.M512365200.

Huelsenbeck JP, Ronquist F. 2001. MRBAYES: Bayesian inference of phylogenetic trees. Bioinformatics 17(8):754-755 DOI 10.1093/bioinformatics/17.8.754.

Kadowaki T, Yukitake H, Naito M, Sato K, Kikuchi Y, Kondo Y, Shoji M, Nakayama K. 2016. A two-component system regulates gene expression of the type IX secretion component proteins via an ECF sigma factor. Scientific Reports 6:23288 DOI $10.1038 /$ srep23288.

Karlsson FH, Ussery DW, Nielsen J, Nookaew I. 2011. A closer look at Bacteroides: phylogenetic relationship and genomic implications of a life in the human gut. Microbial Ecology 61(3):473-485 DOI 10.1007/s00248-010-9796-1.

Katoh K, Misawa K, Kuma KI, Miyata T. 2002. MAFFT: a novel method for rapid multiple sequence alignment based on fast Fourier transform. Nucleic Acids Research 30(14):30593066 DOI 10.1093/nar/gkf436.

Khader YS, Dauod AS, El-Qaderi SS, Alkafajei A, Batayha WQ. 2006. Periodontal status of diabetics compared with nondiabetics: a meta-analysis. Journal of Diabetes and its Complications 20(1):59-68 DOI 10.1016/j.jdiacomp.2005.05.006.

Kinane DF, Stathopoulou PG, Papapanou PN. 2017. Periodontal diseases. Nature Reviews Disease Primers 3:17038 DOI doi:10.1038/nrdp.2017.38.

Lasica AM, Goulas T, Mizgalska D, Zhou X, De Diego I, Ksiazek M, Madej M, Guo Y, Guevara T, Nowak M, Potempa B, Goel A, Sztukowska M, Prabhakar AT, Bzowska M, Widziolek M, Thøgersen IB, Enghild JJ, Simonian M, Kulczyk AW, Nguyen KA, Potempa J, Gomis-Rüth FX. 2016. Structural and functional probing of PorZ, an essential bacterial surface component of the type-IX secretion system of human oral-microbiomic Porphyromonas gingivalis. Scientific Reports 6:37708 DOI 10.1038/srep37708.

Lasica AM, Ksiazek M, Madej M, Potempa J. 2017. The type IX secretion system (T9SS): highlights and recent insights into its structure and function. Frontiers in Cellular and Infection Microbiology 7:215 DOI 10.3389/fcimb.2017.00215.

Lauber F, Deme JC, Lea SM, Berks BC. 2018. Type 9 secretion system structures reveal a new protein transport mechanism. Nature 564(7734):77 DOI 10.1038/s41586-018-0693-y.

Laugisch O, Wong A, Sroka A, Kantyka T, Koziel J, Neuhaus K, Sculean A, Venables PJ, Potempa J, Möller B, Eick S. 2016. Citrullination in the periodontium - a possible link between periodontitis and rheumatoid arthritis. Clinical Oral Investigations 20(4):675-683 DOI 10.1007/s00784-015-1556-7.

Letunic I, Bork P. 2019. Interactive Tree Of Life (iTOL) v4: recent updates and new developments. Nucleic Acids Research 47(W1):W256-W259 DOI 10.1093/nar/gkz239.

Li N, Zhu Y, LaFrentz BR, Evenhuis JP, Hunnicutt DW, Conrad RA, Barbier P, Gullstrand CW, Roets JE, Powers JL, Kulkarni SS, Erbes DH, Garcia JC, Nie P, McBride MJ. 2017. The type IX secretion system is required for virulence of the fish pathogen Flavobacterium columnare. Applied and Environmental Microbiology 83(23):e01769-17 DOI 10.1128/aem.01769-17.

Mahillon J, Chandler M. 1998. Insertion sequences. Microbiology and Molecular Biology Reviews 62(3):725-774 
687

688

689

690

691

692

693

694

695

696

697

698

699

700

701

702

703

704

705

706

707

708

709

710

711

712

713

714

715

716

717

718

719

720

721

722

723

724

725

726

727

728

729

730

731

732

Maresz KJ, Hellvard A, Sroka A, Adamowicz K, Bielecka E, Koziel J, Gawron K, Mizgalska D, Marcinska KA, Benedyk M, Pyrc K, Quirke AM, Jonsson R, Alzabin S, Venables PJ, Nguyen KA, Mydel P, Potempa J. 2013. Porphyromonas gingivalis facilitates the development and progression of destructive arthritis through its unique bacterial peptidylarginine deiminase (PAD). PLoS Pathogens 9(9):e1003627 DOI 10.1371/journal.ppat.1003627.

McBride MJ, Zhu Y. 2013. Gliding motility and Por secretion system genes are widespread among members of the phylum Bacteroidetes. Journal of Bacteriology 195(2):270-278 DOI 10.1128/JB.01962-12.

Miller MA, Pfeiffer W, Schwartz T. 2010. Creating the CIPRES Science Gateway for inference of large phylogenetic trees. In: Proceedings of the Gateway Computing Environments Workshop (GCE). 1-8 DOI 10.1109/GCE.2010.5676129.

Naas AE, Solden LM, Norbeck AD, Brewer H, Hagen LH, Heggenes IM, McHardy AC, Mackie RI, Paša-Tolic L, Arntzen MØ, Eijsink VGH, Koropatkin NM, Hess M, Wrighton KC, Pope PB. 2018. "Candidatus Paraporphyromonas polyenzymogenes" encodes multi-modular cellulases linked to the type IX secretion system. Microbiome 6(1):44 DOI 10.1186/s40168-018-0421-8.

Naito M, Tominaga T, Shoji M, Nakayama K. 2019. PGN_0297 is an essential component of the type IX secretion system (T9SS) in Porphyromonas gingivalis: Tn-seq analysis for exhaustive identification of T9SS-related genes. Microbiology and Immunology 63(1):1120 DOI 10.1111/1348-0421.12665.

Naito M, Hirakawa H, Yamashita A, Ohara N, Shoji M, Yukitake H, Nakayama K, Toh H, Yoshimura F, Kuhara S, Hattori M. 2008. Determination of the genome sequence of Porphyromonas gingivalis strain ATCC 33277 and genomic comparison with strain W83 revealed extensive genome rearrangements in $P$. gingivalis. DNA Research 15(4):215-225 DOI 10.1093/dnares/dsn013.

Nakane D, Sato K, Wada H, McBride MJ, Nakayama K. 2013. Helical flow of surface protein required for bacterial gliding motility. Proceedings of the National Academy of Sciences 110(27):11145-11150 DOI 10.1073/pnas.1219753110.

Nguyen KA, Zylicz J, Szczesny P, Sroka A, Hunter N, Potempa J. 2009. Verification of a topology model of PorT as an integral outer-membrane protein in Porphyromonas gingivalis. Microbiology (Reading, England) 155(Pt 2):328 DOI 10.1099/mic.0.024323-0.

Nikaido H. 2003. Molecular basis of bacterial outer membrane permeability revisited. Microbiology and Molecular Biology Reviews 67(4):593-656 DOI 10.1128/MMBR.67.4.593.

Osbourn AE, Field B. 2009. Operons. Cellular and Molecular Life Sciences 66(23):3755-3775 DOI 10.1007/s00018-009-0114-3.

Pei AY, Oberdorf WE, Nossa CW, Agarwal A, Chokshi P, Gerz EA, Jin Z, Lee P, Yang L, Poles M, Brown SM, Sotero S, DeSantis T, Brodie E, Nelson K, Pei Z. 2010. Diversity of 16S rRNA genes within Individual Prokaryotic Genomes. Applied and Environmental Microbiology 76(12):3886-3897 DOI 10.1128/AEM.02953-09.

Potempa J, Pike R, Travis J. 1995. The multiple forms of trypsin-like activity present in various strains of Porphyromonas gingivalis are due to the presence of either Arg-gingipain or Lys-gingipain. Infection and Immunity 63(4):1176-1182

Pylro VS, Vespoli LDS, Duarte GF, Yotoko KSC. 2012. Detection of horizontal gene transfers from phylogenetic comparisons. International Journal of Evolutionary Biology 2012 DOI

PeerJ reviewing PDF | (2019:06:38397:4:0:NEW 27 Mar 2020) 
733

734

735

736

737

738

739

740

741

742

743

744

745

746

747

748

749

750

751

752

753

754

755

756

757

758

759

760

761

762

763

764

765

766

767

768

769

770

771

772

773

774

775

776

777

778

$10.1155 / 2012 / 813015$.

Rahman MS, Simser JA, Macaluso KR, Azad AF. 2003. Molecular and functional analysis of the lepB gene, encoding a type I signal peptidase from Rickettsia rickettsii and Rickettsia typhi. Journal of Bacteriology 185(15):4578-4584 DOI 10.1128/JB.185.15.45784584.2003.

Saiki K, Konishi K. 2010. Identification of a novel Porphyromonas gingivalis outer membrane protein, PG534, required for the production of active gingipains. FEMS Microbiology Letters 310(2):168-174 DOI 10.1111/j.1574-6968.2010.02059.x.

Sato K, Naito M, Yukitake H, Hirakawa H, Shoji M, McBride MJ, Rhodes RG, Nakayama K. 2010. A protein secretion system linked to bacteroidete gliding motility and pathogenesis. Proceedings of the National Academy of Sciences 107(1):276-281 DOI 10.1073/pnas.0912010107.

Schwarz G. 1978. Estimating the dimension of a model. The Annals of Statistics 6(2):461-464

Sela I, Ashkenazy H, Katoh K, Pupko T. 2015. GUIDANCE2: Accurate detection of unreliable alignment regions accounting for the uncertainty of multiple parameters. Nucleic Acids Research 43(W1):W7-W14 DOI 10.1093/nar/gkv318.

Shoji M, Sato K, Yukitake H, Kamaguchi A, Sasaki Y, Naito M, Nakayama K. 2018. Identification of genes encoding glycosyltransferases involved in lipopolysaccharide synthesis in Porphyromonas gingivalis. Molecular Oral Microbiology 33(1):68-80 DOI 10.1111/omi.12200.

Shoji M, Sato K, Yukitake H, Naito M, Nakayama K. 2014. Involvement of the Wbp pathway in the biosynthesis of Porphyromonas gingivalis lipopolysaccharide with anionic polysaccharide. Scientific Reports 4:5056 DOI 10.1038/srep05056.

Shoji M, Yukitake H, Sato K, Shibata Y, Naito M, Aduse-Opoku J, Abiko Y, Curtis MA, Nakayama K. 2013. Identification of an O-antigen chain length regulator, WzzP, in Porphyromonas gingivalis. MicrobiologyOpen 2(3):383-401 DOI 10.1002/mbo3.84.

Shoji M, Ratnayake DB, Shi Y, Kadowaki T, Yamamoto K, Yoshimura F, Akamine A, Curtis MA, Nakayama K. 2002. Construction and characterization of a nonpigmented mutant of Porphyromonas gingivalis: cell surface polysaccharide as an anchorage for gingipains. Microbiology 148(4):1183-1191 DOI 10.1099/00221287-148-4-1183.

Taguchi Y, Sato K, Yukitake H, Inoue T, Nakayama M, Naito M, Kondo Y, Kano K, Hoshino T, Nakayama K, Takashiba S, Ohara N. 2016. Involvement of an Skp-like protein, PGN_0300, in the Type IX secretion system of Porphyromonas gingivalis. Infection and Immunity 84(1):230-240 DOI 10.1128/IAI.01308-15.

Thomas CM, Nielsen KM. 2005. Mechanisms of, and barriers to, horizontal gene transfer between bacteria. Nature Reviews Microbiology 3(9):711 DOI 10.1038/nrmicro1234.

Vincent MS, Chabalier M, Cascales E. 2018. A conserved motif of Porphyromonas Type IX secretion effectors C-terminal secretion signal specifies interactions with the PorKLMN core complex. BioRxiv:483123 DOI 10.1101/483123.

Vincent MS, Canestrari MJ, Leone P, Stathopulos J, Ize B, Zoued A, Cambillau C, Kellenberger C, Roussel A, Cascales E. 2017. Characterization of the Porphyromonas gingivalis type IX secretion trans-envelope PorKLMNP core complex. Journal of Biological Chemistry 292(8):3252-3261 DOI 10.1074/jbc.M116.765081.

Winker S, Woese CR. 1991. A Definition of the Domains Archaea, Bacteria and Eucarya in Terms of Small Subunit Ribosomal RNA Characteristics. Systematic and Applied Microbiology 14(4):305-310 DOI 10.1016/S0723-2020(11)80303-6.

Peer) reviewing PDF | (2019:06:38397:4:0:NEW 27 Mar 2020) 
779 Yanofsky C, Lennox ES. 1959. Transduction and recombination study of linkage relationships 780 among the genes controlling tryptophan synthesis in Escherichia coli. Virology 8(4):425$781 \quad 447$ DOI 10.1016/0042-6822(59)90046-7. 
Figure 1

T9SS protein components on the inner membrane (IM) and outer membrane (OM) of Porphyromonas gingivalis.

The protein components with known functions are represented by coloured structures. The pathway for cargo protein gingipain (RgpB) translocation and modifications by T9SS is illustrated. The regulation of the pathway by the protein components is also exhibited.

\section{Extracellular milieu}

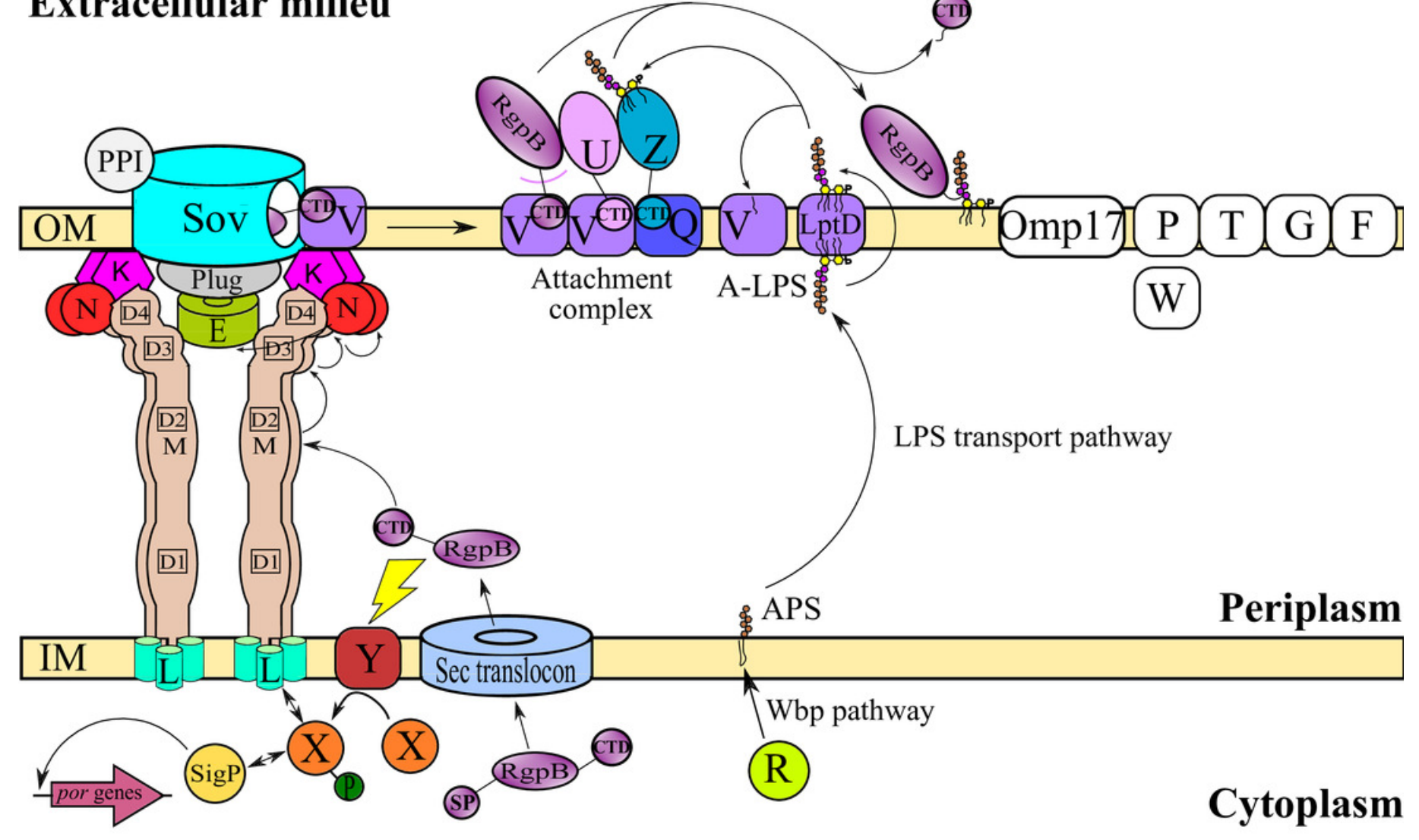




\section{Figure 2}

The Bayesian Inference (BI) phylogenetic trees of T9SS protein components (PorK, PorL, PorM, and PorN).

(A) BI tree of Pork. (B) BI tree of PorL. (C) BI tree of PorM. (D) BI tree of PorN. The terminal nodes are labelled with coloured circles that represent the classes under Bacteroidetes that each protein homolog belongs to. The classes represented by each colour are provided in the legend inside the figure. The branches with strong support (posterior probability value > 0.95) are coloured in black. Otherwise, the branches are coloured in red. The solid curve denotes a monophyletic clade that was formed by terminal nodes that belong to the same class under Bacteroidetes. The dashed curve denotes a strong support for the monophyletic clade or its subclade. The colour of curve represents the class of terminal nodes that form the clade. The classes represented by each colour are shown in the legend inside the figure. 
A

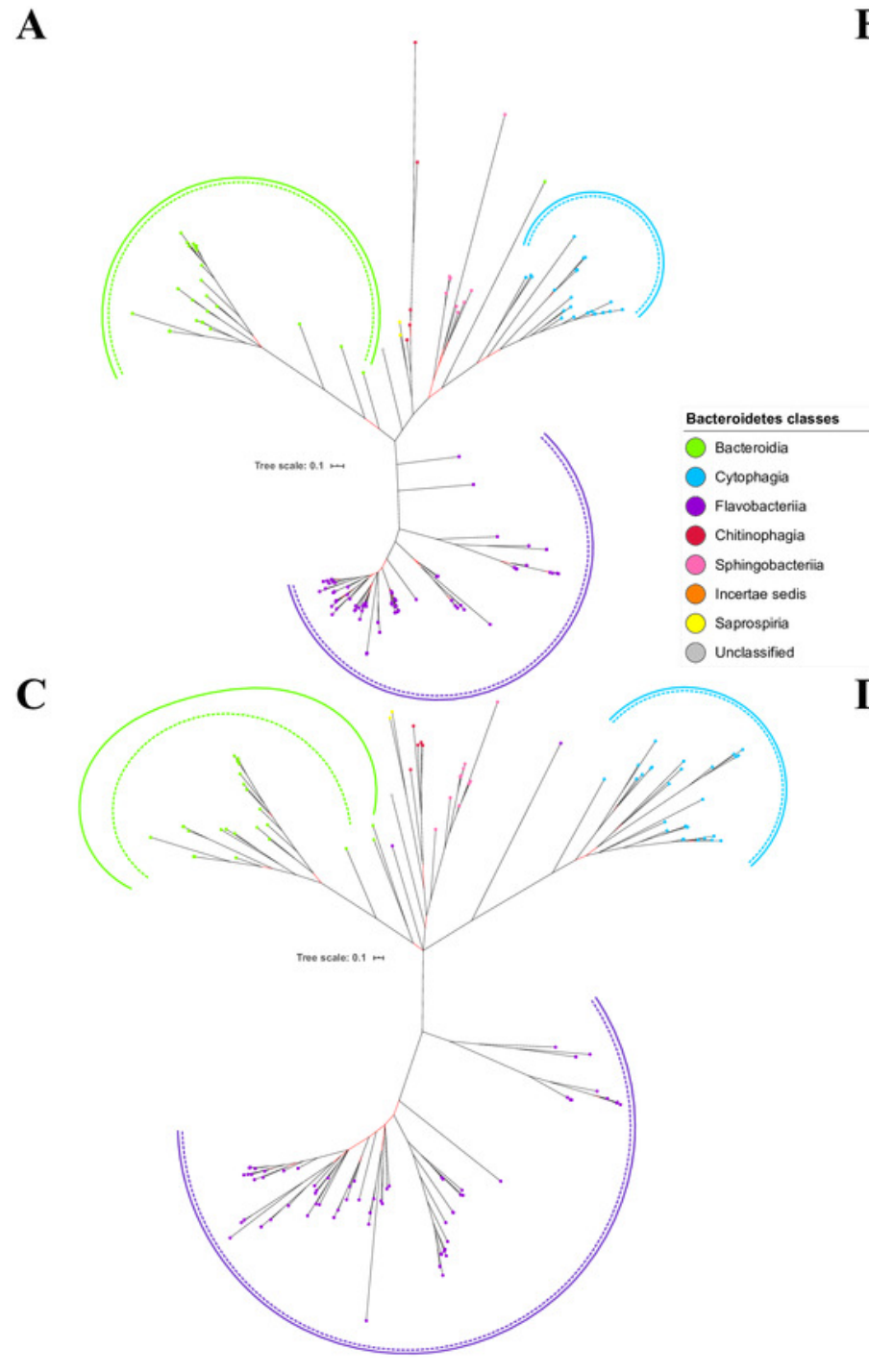

B

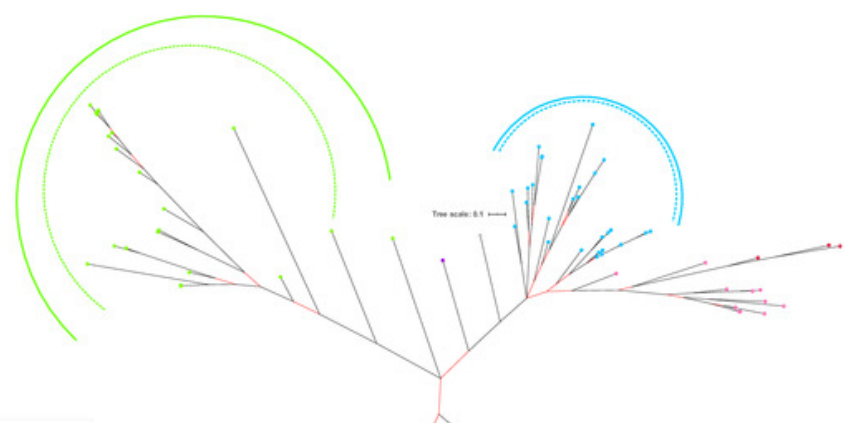

D

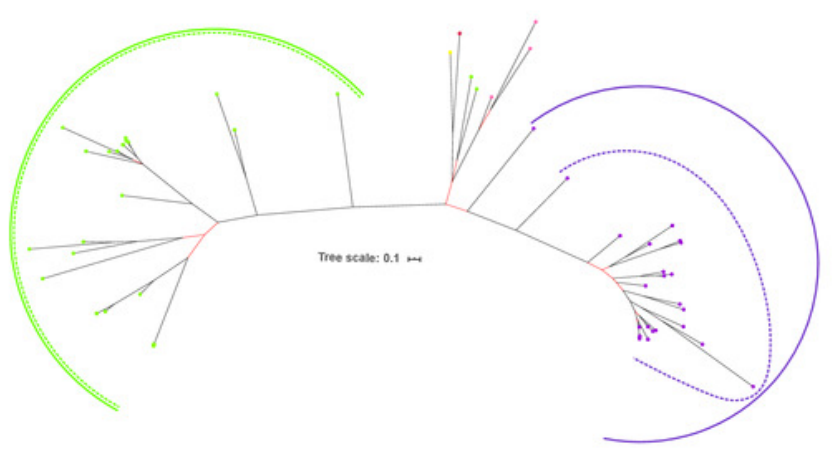


Figure 3

The Bayesian Inference (BI) phylogenetic trees of T9SS protein components (PorP, PorQ, PorR, and Sov).

(A) BI tree of PorP. (B) BI tree of PorQ. (C) BI tree of PorR. (D) BI tree of Sov.

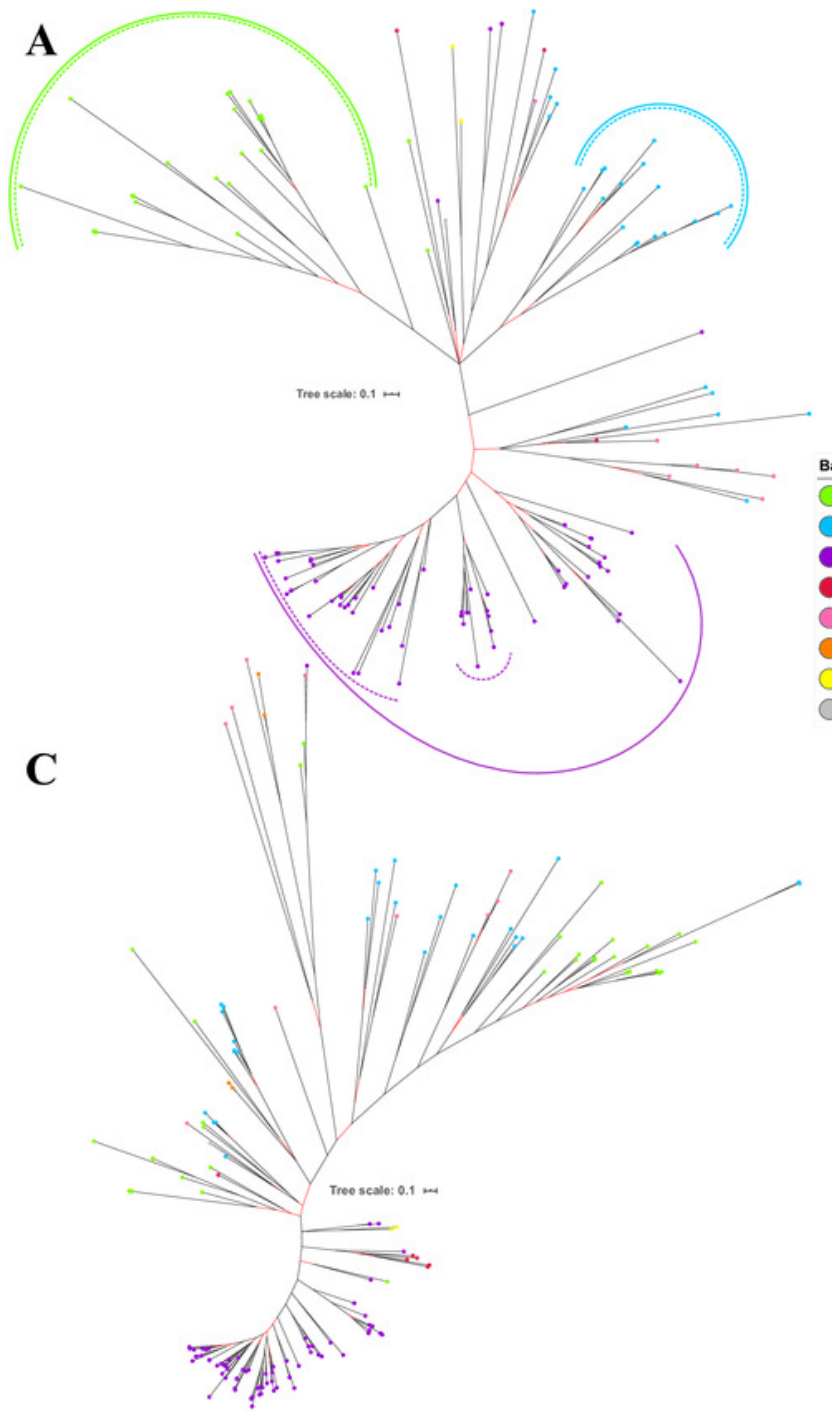

B
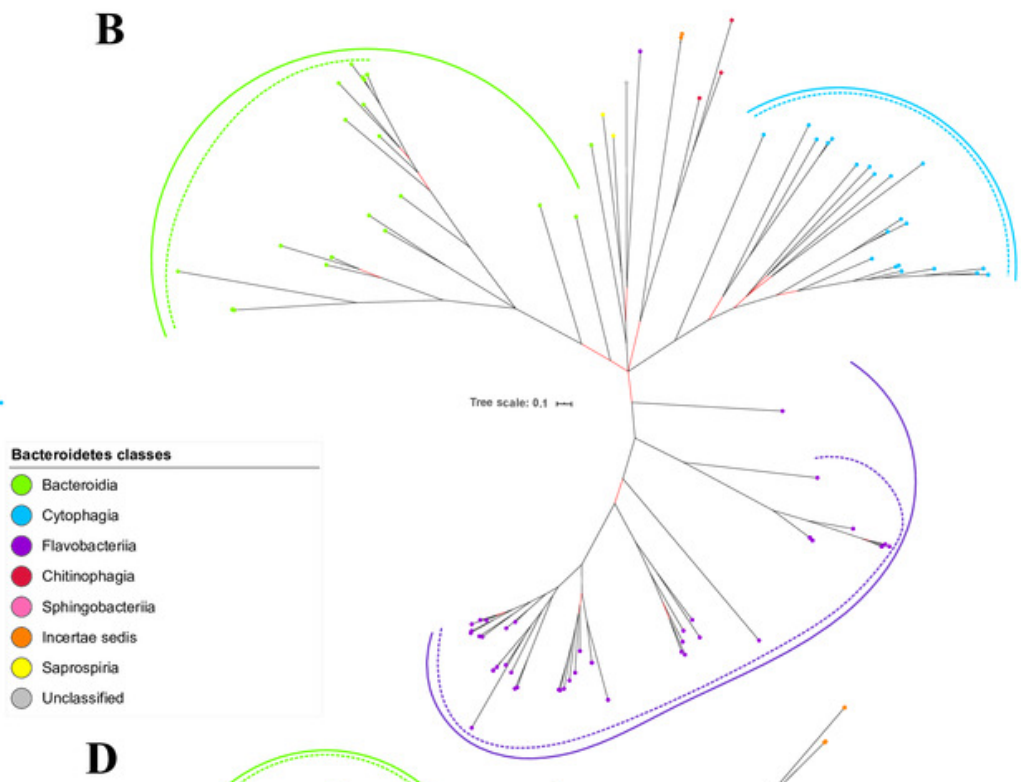

D

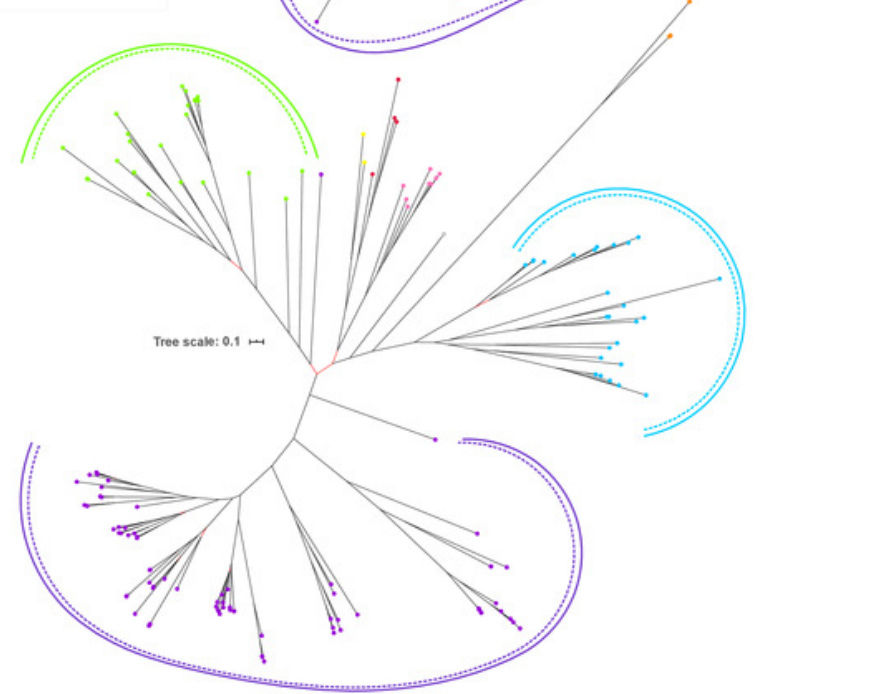


Figure 4

The Bayesian Inference (BI) phylogenetic trees of T9SS protein components (PorT, PorU, PorV, and PorW).

(A) BI tree of PorT. (B) BI tree of PorU. (C) BI tree of PorV. (D) BI tree of PorW.

A

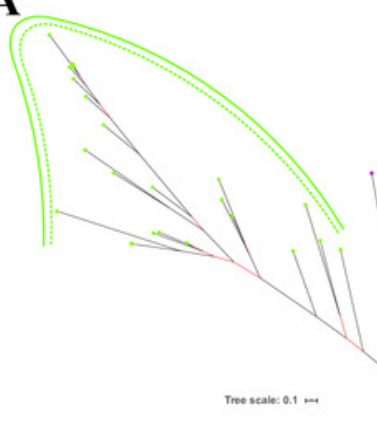

C
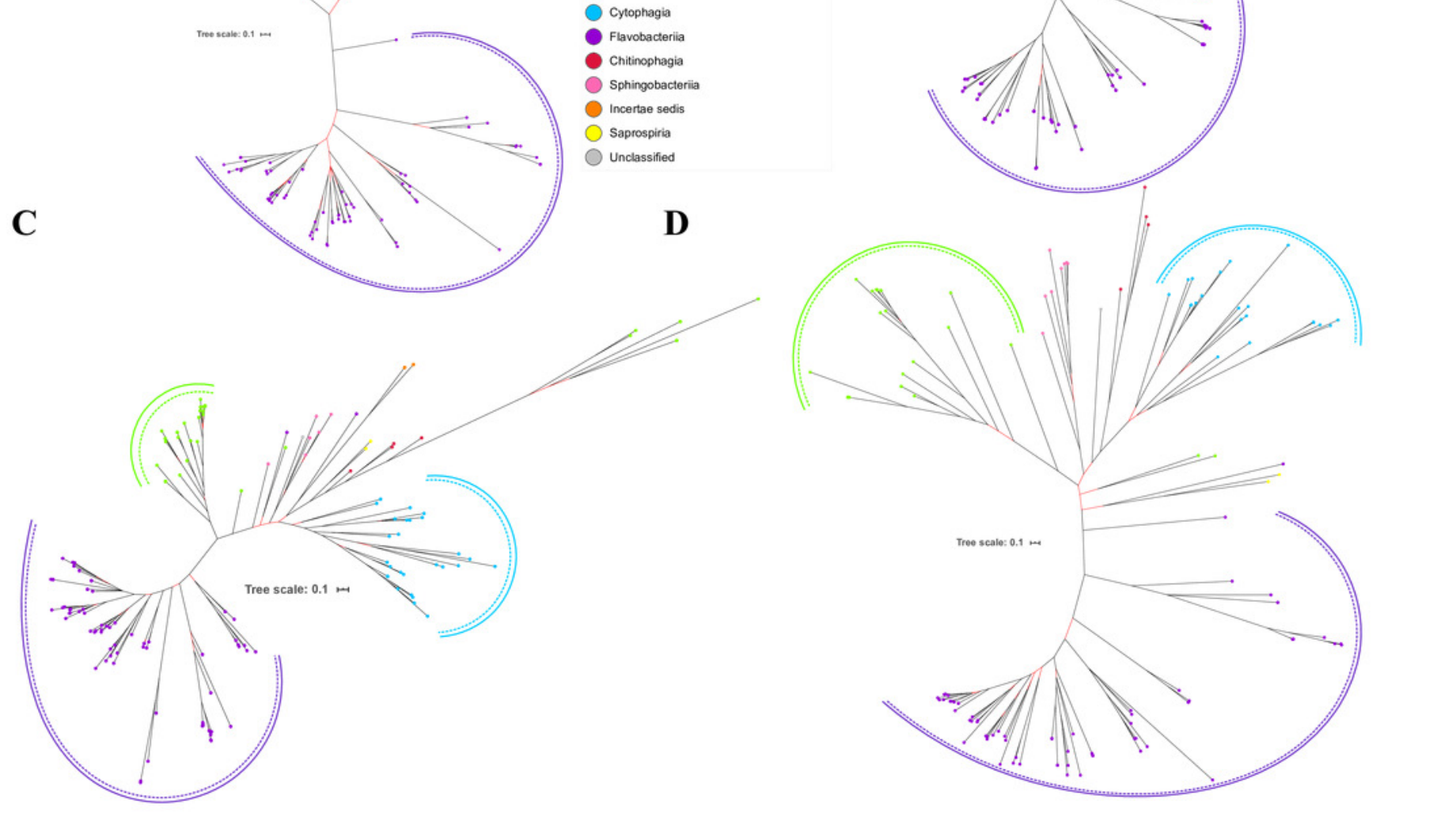

B

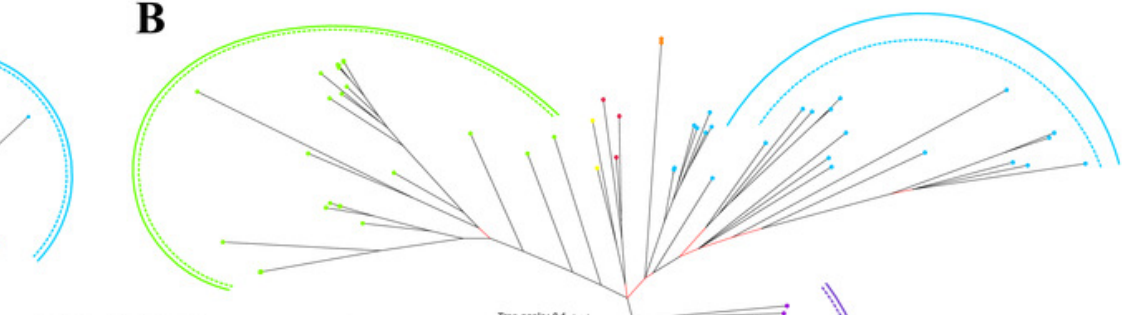


Figure 5

The Bayesian Inference (BI) phylogenetic trees of T9SS protein components (PorX, PorY, PorZ, and SigP).

(A) BI tree of PorX. (B) BI tree of PorY. (C) BI tree of PorZ. (D) BI tree of SigP.

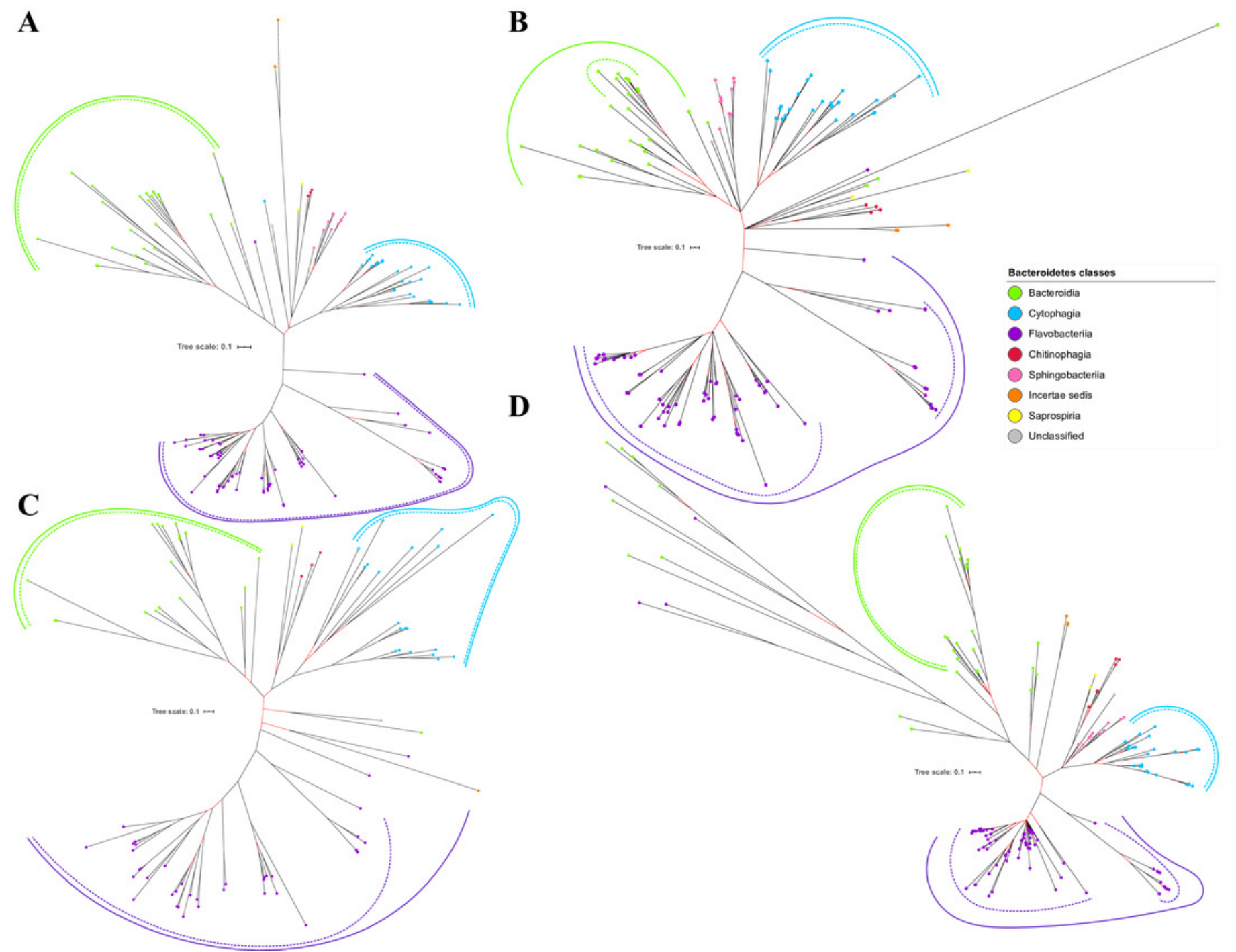


Figure 6

The Bayesian Inference (BI) phylogenetic trees of T9SS protein components (Omp17, PorE, PorF, and PorG).

(A) BI tree of Omp17. (B) BI tree of PorE. (C) BI tree of PorF. (D) BI tree of PorG.
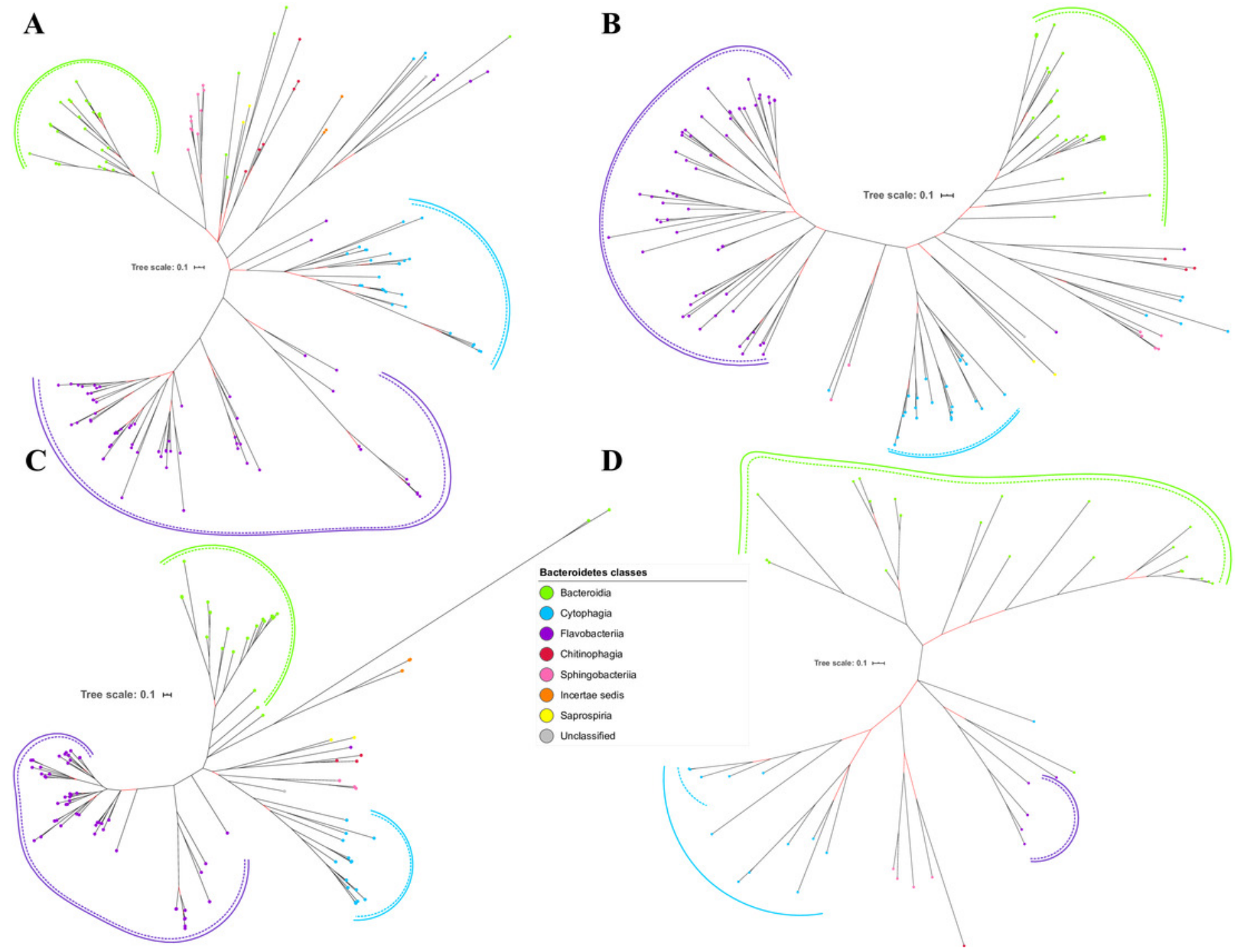


\section{Figure 7}

The Bayesian Inference (BI) phylogenetic tree of T9SS containing Bacteroidetes species $16 \mathrm{~S}$ ribosomal RNA ( $\mathrm{RNNA}$ ).

The BI tree of 16S rRNA exhibits monophyletic clades where each clade consists of terminal nodes of the same colour that denotes that they belong to the same class under Bacteroidetes. There is a high support (posterior probability value $>0.95$ ) for each monophyletic clade indicates by the black branch leading to each clade. The solid and dashed green, purple, and blue curves indicate there is a strong support for the monophyletic clades of Bacteroidia, Flavobacteriia, and Cytophagia classes respectively. 


\begin{tabular}{l} 
Bacteroidetes classes \\
\hline Bacteroidia \\
Cytophagia \\
Flavobacteriia \\
Chitinophagia \\
Sphingobacteriia \\
Incertae sedis \\
Saprospiria \\
Unclassified
\end{tabular}

Tree scale: 1

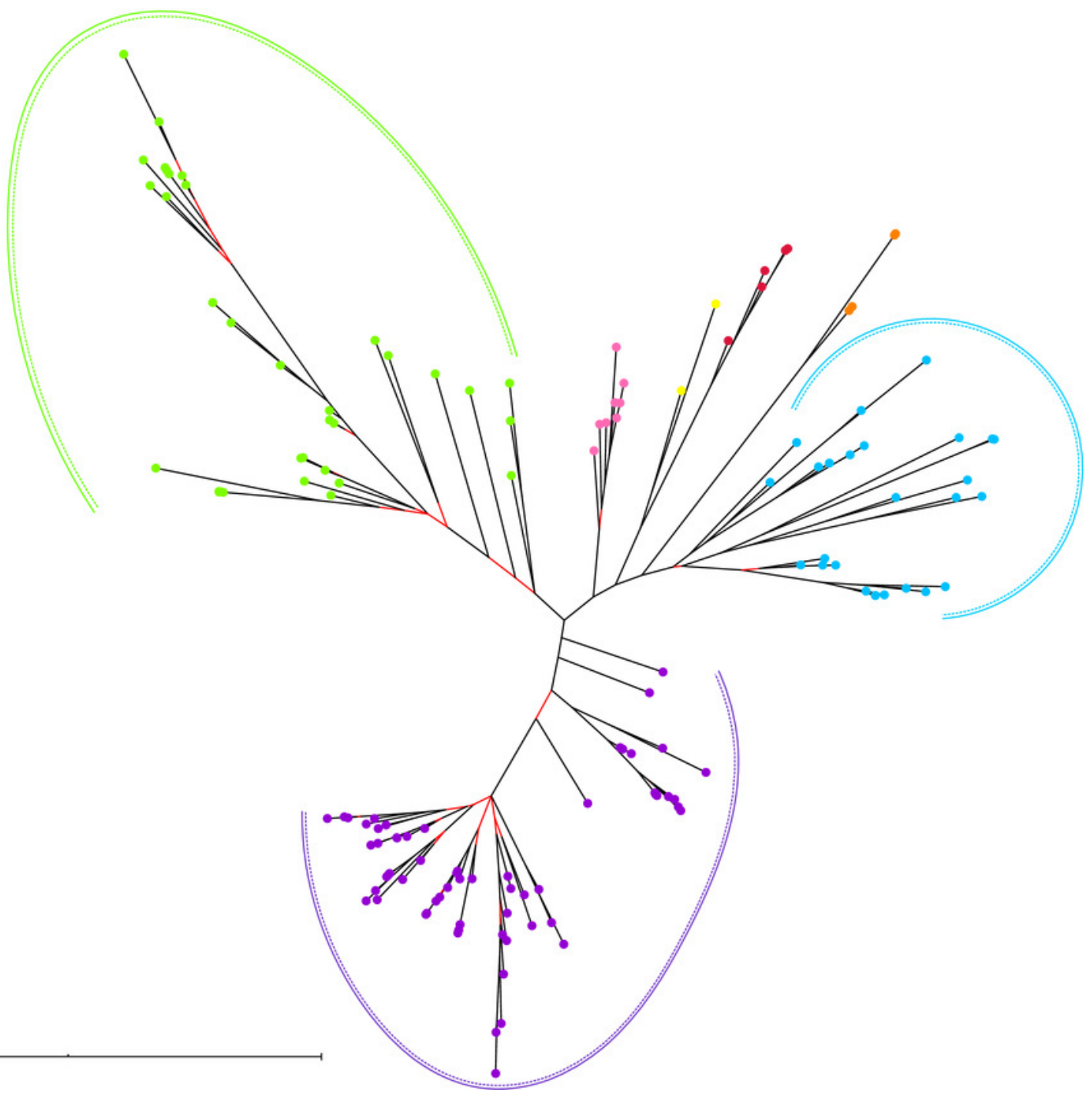




\section{Figure 8}

The arrangement of porR and its neighbouring genes in P. gingivalis ATCC 33277 genome.

porR (PGN_1236) and its neighbouring genes are flanked by IS5 family transposons that formed a composite transposon of $70 \mathrm{kbp}$ in length. The genes that involve in biosynthesis of A-LPS are represented by yellow rectangles while the gene that does not involve is represented by brown rectangle. The genes for hypothetical proteins are represented by white rectangles. The genes for IS5 family transposases are represented by cyan rectangles. The purple triangles represented 12 bp inverted repeats that flanked the genes for IS5 family transposases. Name of proteins encoded by the genes are shown under rectangles that represented the genes. The slashes indicated gaps in the genome. 

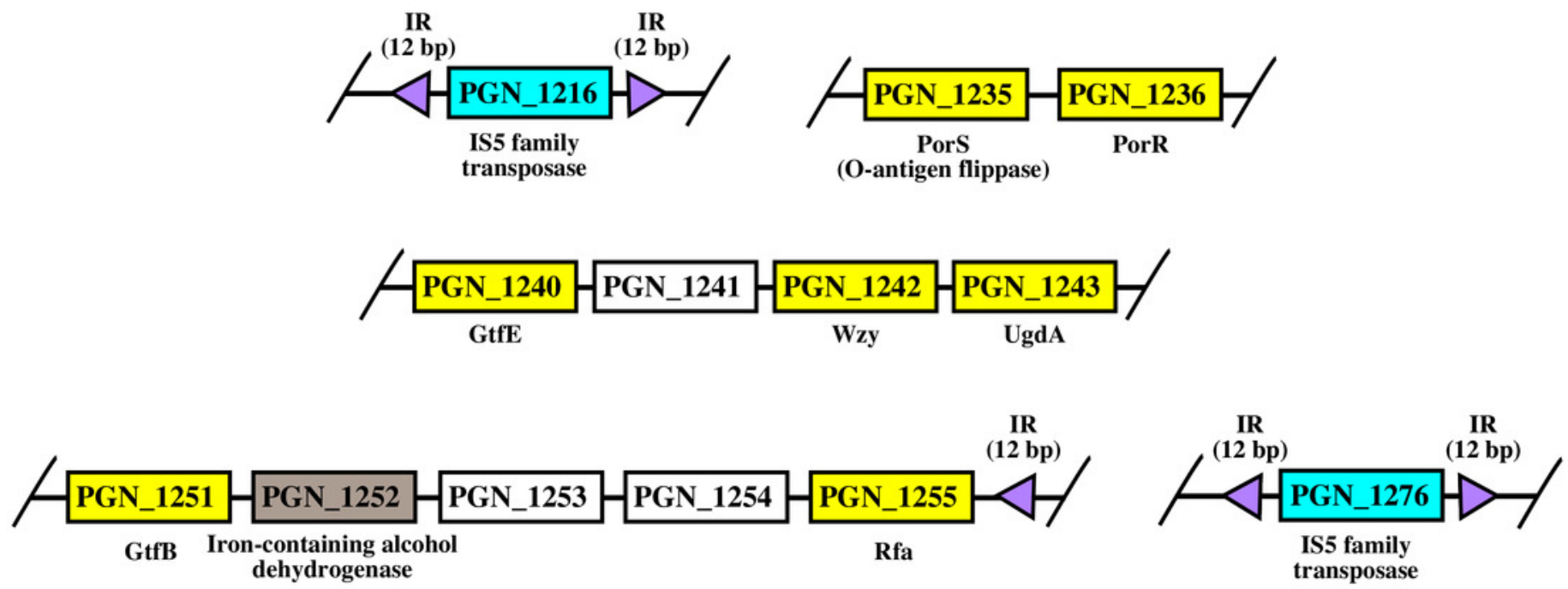


\section{Figure 9}

Comparison between the Bayesian Inference (BI) tree of UgdA with BI tree of PorR.

The BI tree of UgdA (A) does not exhibit monophyletic clades for all major classes under

Bacteroidetes which is similar to the BI tree of PorR (B). Both BI trees of UgdA and PorR also exhibit similar topology. Both trees exhibit cluster I (solid purple curve) that primarily consists of terminal nodes of Flavobacteriia and a few terminal nodes from other classes. Both trees have cluster II (solid green curve) that consists of terminal nodes of Porphyromonas, Tannerella, and Parabacteroides genera. Both trees acquire cluster III (solid blue curve) that consists of terminal nodes of Rufibacter and Hymenobacter genera. Both trees exhibit cluster IV (solid green curve) that consists of terminal nodes of Prevotella, Bacteroides, Proteiniphilum, and other genera. 


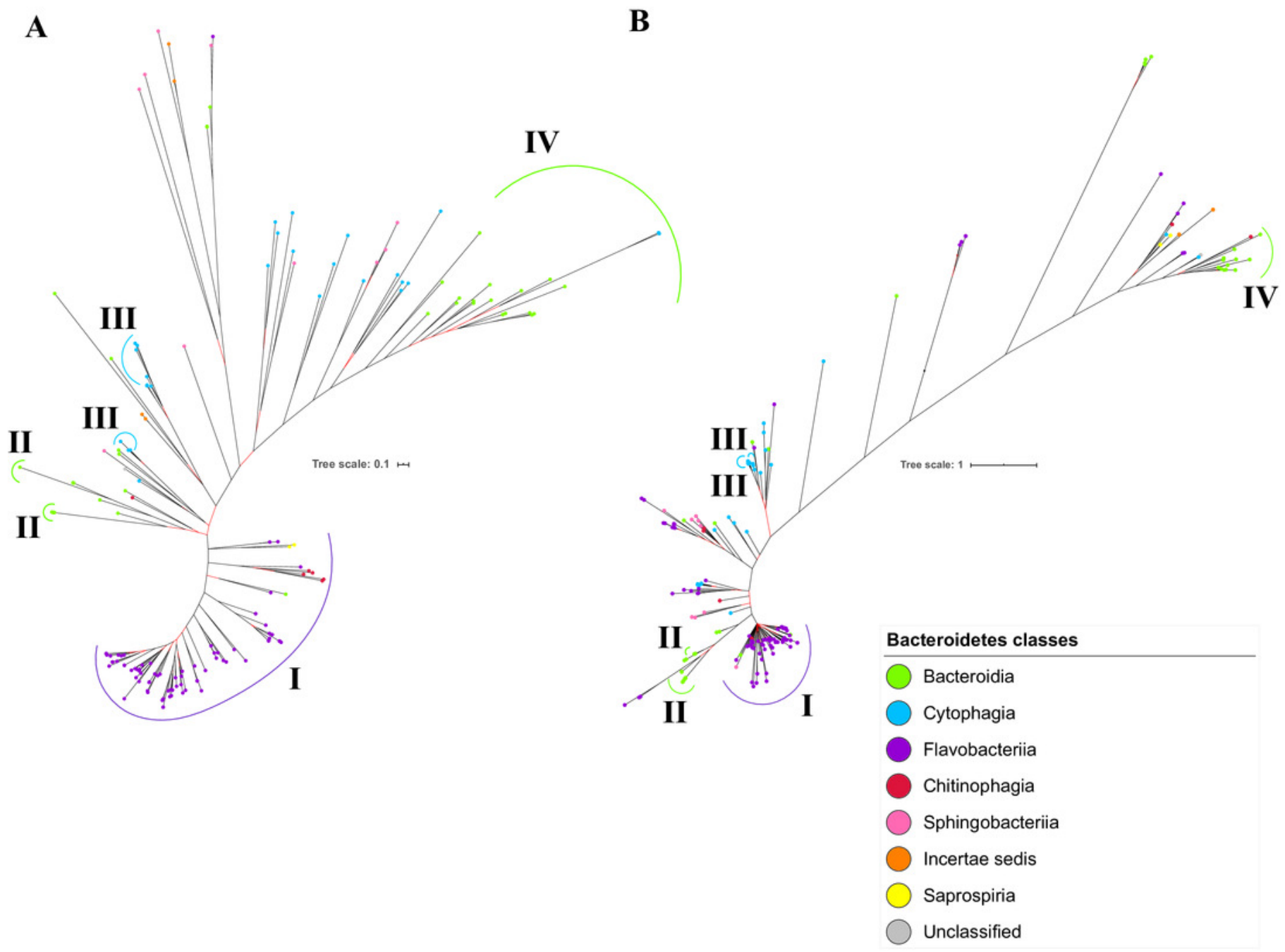




\section{Figure 10}

The species from Chitinophagia, Saprospiria, and unclassified under Bacteroidetes phylum that acquired homologs of T9SS protein components.

The colours of rectangles denote the classes those species belong to. Coloured squares indicate T9SS component homologs acquired by the species where the different colours denote different functions those components performed. White squares indicate T9SS component homologs absent in those species.

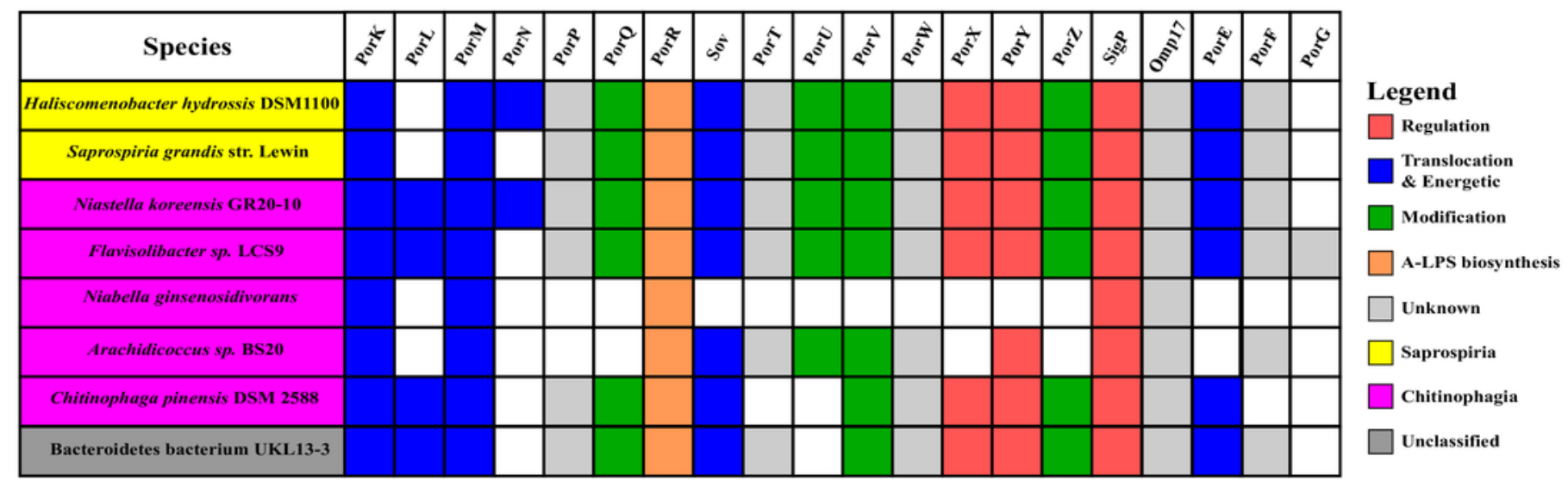




\section{Table $\mathbf{1}$ (on next page)}

The characteristics of T9SS component protein alignments and the best amino acid substitution models that have been selected for them.

The characteristics of T9SS component protein alignments such as no. of taxa used to construct the alignments and no. of characters of the alignments are provided. The best amino acid substitution model that has been selected for each alignment is also provided. The definition of parameters of the best amino acid substitution models are provided in the footnote. 


\begin{tabular}{|l|l|l|l|}
\hline Alignment & No. of taxa & No. of characters & Model \\
\hline Omp17 & 180 & 245 & LG + G + F \\
\hline PorE & 137 & 793 & LG + G + I \\
\hline PorF & 121 & 829 & LG + G + I + F \\
\hline PorG & 55 & 487 & LG + G + I \\
\hline PorK & 153 & 561 & LG + G + I \\
\hline PorL & 123 & 281 & LG + G + I \\
\hline PorM & 159 & 406 & LG + G + I + F \\
\hline PorN & 62 & 267 & LG + G + I \\
\hline PorP & 138 & 281 & LG + G + I + F \\
\hline PorQ & 108 & 358 & LG + G + F \\
\hline PorR & 176 & 471 & LG + G + I \\
\hline PorT & 151 & 202 & LG + G + F \\
\hline PorU & 109 & 919 & LG + G + I \\
\hline PorV & 162 & 360 & LG + G + I + F \\
\hline PorW & 137 & 995 & LG + G + I + F \\
\hline PorX & 162 & 624 & LG + G + I \\
\hline PorY & 162 & 897 & LG + G + I \\
\hline PorZ & 102 & 569 & LG + G + I \\
\hline SigP & 177 & 197 & LG + G + I \\
\hline Sov & 159 & 2704 & LG + G + I + F \\
\hline UgdA & 176 & 460 & LG + G + I \\
\hline $16 S$ rRNA & 144 & 1452 & \\
\hline Not? & & & \\
\hline
\end{tabular}

- $\quad$ LG + G: LG substitution model matrix with gamma-shaped rate variation across sites and Dirichlet stationary amino acid frequencies

- $\quad \mathrm{LG}+\mathrm{G}+\mathrm{I}$ : $\mathrm{LG}$ substitution model matrix with gamma-shaped rate variation across sites with a proportion of invariable sites and Dirichlet stationary amino acid frequencies

- $\quad \mathrm{LG}+\mathrm{G}+\mathrm{I}+\mathrm{F}$ : $\mathrm{LG}$ substitution model matrix with gamma-shaped rate variation across sites with a proportion of invariable sites and fixed (empirical) stationary amino acid frequencies

- $\mathrm{LG}+\mathrm{G}+\mathrm{F}$ : $\mathrm{LG}$ substitution model matrix with gamma-shaped rate variation across sites and fixed (empirical) stationary amino acid frequencies

- $\quad$ GTR + G + I: General Time Reversible (GTR) substitution model matrix with gamma-shaped rate variation across sites with a proportion of invariable sites and Dirichlet stationary nucleotide frequencies 